\title{
A new SNOW stream cipher called SNOW-V
}

\author{
Patrik Ekdahl $^{1}$, Thomas Johansson ${ }^{2}$, Alexander Maximov ${ }^{1}$ and Jing Yang ${ }^{2}$ \\ 1 Ericsson Research, Lund, Sweden \\ \{patrik.ekdahl, alexander.maximov\}@ericsson.com \\ ${ }^{2}$ Dept. of Electrical and Information Technology, Lund University, Lund, Sweden \\ \{thomas.johansson, jing.yang\}@eit.Ith.se
}

\begin{abstract}
In this paper we are proposing a new member in the SNOW family of stream ciphers, called SNOW-V. The motivation is to meet an industry demand of very high speed encryption in a virtualized environment, something that can be expected to be relevant in a future $5 \mathrm{G}$ mobile communication system. We are revising the SNOW 3G architecture to be competitive in such a pure software environment, making use of both existing acceleration instructions for the AES encryption round function as well as the ability of modern CPUs to handle large vectors of integers (e.g. SIMD instructions). We have kept the general design from SNOW 3G, in terms of linear feedback shift register (LFSR) and Finite State Machine (FSM), but both entities are updated to better align with vectorized implementations. The LFSR part is new and operates 8 times the speed of the FSM. We have furthermore increased the total state size by using 128-bit registers in the FSM, we use the full AES encryption round function in the FSM update, and, finally, the initialization phase includes a masking with key bits at its end. The result is an algorithm generally much faster than AES-256 and with expected security not worse than AES-256.
\end{abstract}

Keywords: SNOW · Stream Cipher · 5G Mobile System Security.

\section{Introduction}

Stream ciphers have always played an important part in securing the various generations of 3GPP mobile telephony systems, starting with the GSM system employing the A5 suit of ciphers, continuing with the use of SNOW $3 \mathrm{G}$ as one of the core algorithm for integrity and confidentiality in both UMTS and LTE. When we now turn to the next generation system, called $5 \mathrm{G}$, we see some fundamental changes in system architecture and security level that in many cases invalidate the previous algorithms. We will focus on the LTE (or $4 \mathrm{G}$, as it is commonly called) system when describing the current state in link protection for mobile systems.

The basis for the link security in all 3GPP generations of mobile telephony systems is a shared secret key between the device (commonly called the User Equipment, UE) and the home network, the Mobile Network Operator that the user has a service agreement with, and from whom the user receives the credentials in form of a UICC with a USIM application (often referred to as the SIM-card). The shared key is stored in the Home Subscriber Server (HSS) and in the Secure Element on the UICC. From this key, through a set of key derivations, the home network and the UE both agree on new keys to be used for integrity and confidentiality protection of the control channel, and confidentiality protection of the user data channel. The $4 \mathrm{G}$ system defines three different possible algorithms for integrity (128-EIAx) and confidentiality (128-EEAx), based on three different primitives SNOW 3G [SAG06], AES [oST01], and ZUC [SAG11]. The algorithms used in UMTS and LTE are all using the 128-bit key size, and are depicted in Table 1. 
Table 1: Base algorithms used in UMTS and LTE for integrity and confidentiality.

\begin{tabular}{|l||c|c|c|c|}
\hline \multicolumn{1}{|c||}{} & \multicolumn{2}{c|}{ UMTS } & \multicolumn{2}{c|}{ LTE } \\
& Integrity & Encryption & Integrity & Encryption \\
\hline Kasumi & UIA1 & UEA1 & & \\
SNOW 3G & UIA2 & UEA2 & EIA1 & EEA1 \\
AES & & & EIA2 & EEA2 \\
ZUC & & & EIA3 & EEA3 \\
\hline
\end{tabular}

The SNOW family of stream ciphers started with the SNOW [EJ01] proposal in the European project NESSIE, a call for new primitives. Two attacks [HR02, CHJ02] were soon discovered and the design was subsequently updated to the SNOW 2.0 [EJ02] design. Attacks on SNOW 2.0 will be more discussed in Section 3. The ETSI Security Algorithm Group of Experts (SAGE) modified the SNOW 2.0 design and proposed the resulting cipher SNOW $3 \mathrm{G}$ as one of the algorithms protecting the air interface in 3GPP telecommunication networks.

Although sufficient for $4 \mathrm{G}$ system, these 128-EIAx and 128-EEAx algorithms face some challenges in the $5 \mathrm{G}$ environment. For the $5 \mathrm{G}$ system, the $3 \mathrm{GPP}$ standardization organization is looking towards increasing the security level to 256-bit key lengths [SA318]. For ExA1, and ExA2, this does not immediately appear to be a problem, since both the underlying primitives (AES and SNOW) are specified for 256-bit keys. ZUC is currently only specified and evaluated under 128-bit key strength, but another version, ZUC-256, supporting 256-bit keys has recently been presented [Bin]. However, since the design of the radio and core network will also fundamentally change in the $5 \mathrm{G}$ system, there are other challenges. Many of the network nodes will become virtualized [3GP] and thus the ability to use specialized hardware for the cryptographic primitives will be reduced. Many newer processors from both Intel and ARM now include instructions to accelerate AES, and it will be fairly easy to reach encryption speeds of 20-25 Gbps for EIA2 and EEA2, but for the stream ciphers SNOW and ZUC, we need to look for other solutions. Current benchmarks on SNOW 3G gives approximately 9 Gbps in a pure software implementation, which is far too low for the targeted speed of 20 Gbps downlink in the $5 \mathrm{G}$ system (see, e.g., [ITU17]).

In this paper we revise the SNOW 2.0/ SNOW 3G design to be competitive in a pure software environment, relying on both the acceleration instructions for the AES round function as well as the ability of modern CPUs to handle large vectors of integers (e.g. SIMD instructions). We have kept most of the design from SNOW 3G, in terms of linear feedback shift register (LFSR) and Finite State Machine (FSM), but both entities are updated to better align with vectorized implementations. We have also increased the total state size by going from 32-bit registers to 128-bit registers in the FSM. Each clocking of SNOW-V (V for Virtualization) now produces 128 bits of keystream.

We also propose an AEAD (Authenticated Encryption with Associated Data) operational mode to provide both confidentiality and integrity protection. The keystream width of 128 bits makes the authentication framework of GMAC [Dwo07] very easy to be adopted to SNOW-V.

This paper is organized as follows. In Section 2, we present the new design, including pseudocode. In Section 3 we give a brief security analysis, describing most of the common attack approaches and how they apply to SNOW-V. In Section 4 we describe how authentication can be included in an AEAD mode of operation. Then software implementation aspects are considered in Section 5, and in Section 6 software performance results and implementation aspects using future SIMD instruction set are presented. We end the paper with some conclusions in Section 7. 


\section{The design}

SNOW-V follows the design pattern of previous SNOW versions and consists of an LFSR part and an FSM part. The overall schematic is shown in Figure 1. The LFSR part is now a circular construction consisting of two shift registers, each feeding into the other. The FSM has three 128-bit registers and two instances of a single AES encryption round function.

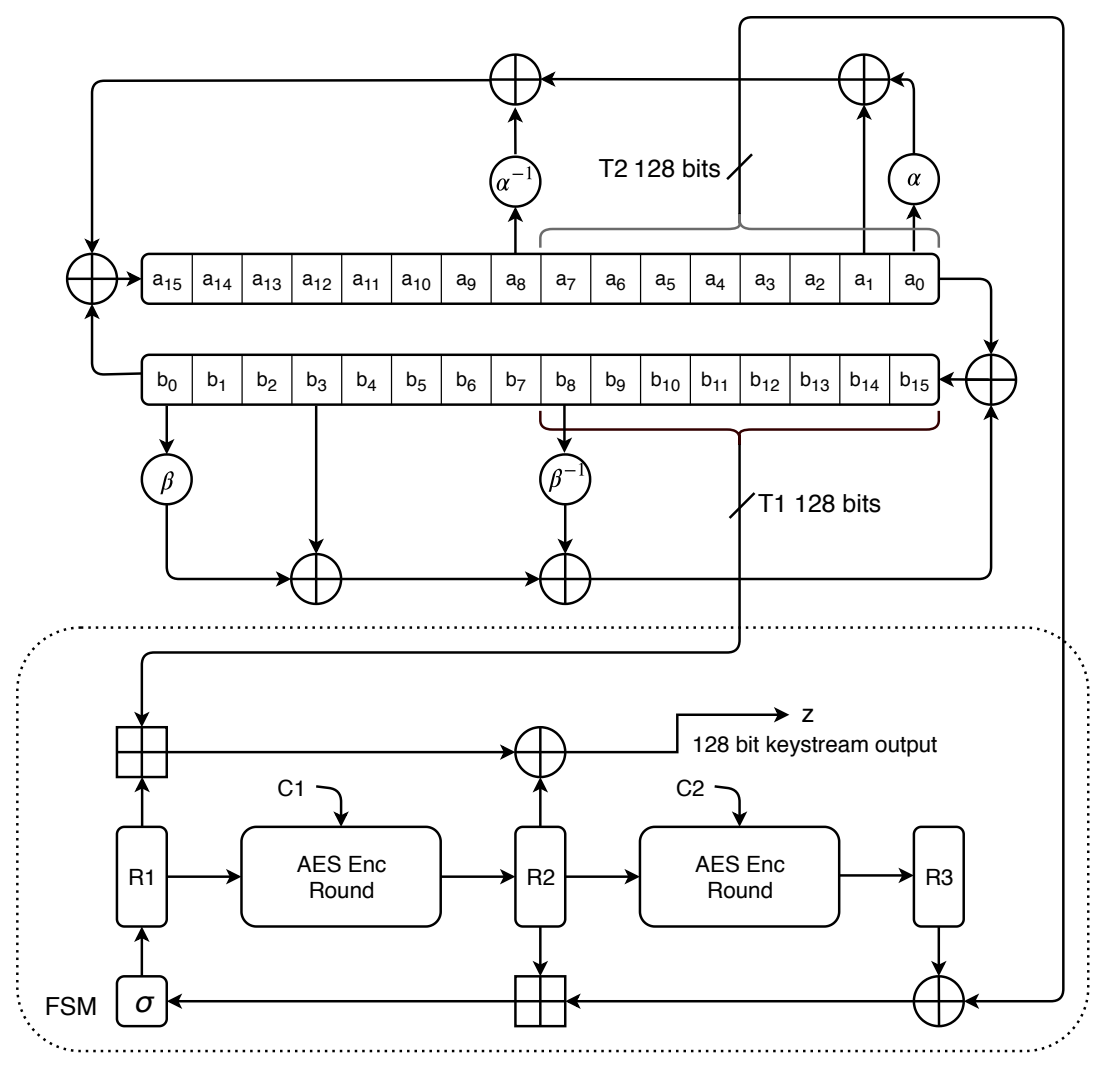

Figure 1: Overall schematics of SNOW-V.

Starting with the LFSR part, we will now provide a detailed description of the design. The two LFSRs are named LFSR-A and LFSR-B, both of length 16 and with a cell size of 16 bits. The 32 cells are denoted $a_{15} \ldots a_{0}$ and $b_{15} \ldots b_{0}$ respectively.

Each cell represents an element in $\mathbb{F}_{2^{16}}$, but LFSR-A and LFSR-B have different generating polynomials. The elements of LFSR-A are generated by the polynomial

$$
g^{A}(x)=x^{16}+x^{15}+x^{12}+x^{11}+x^{8}+x^{3}+x^{2}+x+1 \in \mathbb{F}_{2}[x]
$$

and the elements of LFSR-B are generated by

$$
g^{B}(x)=x^{16}+x^{15}+x^{14}+x^{11}+x^{8}+x^{6}+x^{5}+x+1 \in \mathbb{F}_{2}[x] .
$$

When we consider these elements of $\mathbb{F}_{2^{16}}$ as words, the $x^{0}$ position will be the least significant bit in the word. Let $\alpha \in \mathbb{F}_{2^{16}}^{A}$ be a root of $g^{A}(x)$ and $\beta \in \mathbb{F}_{2^{16}}^{B}$ be a root of $g^{B}(x)$. At time $t \geq 0$ we denote the states of the LFSRs as $\left(a_{15}^{(t)}, a_{14}^{(t)}, \ldots, a_{1}^{(t)}, a_{0}^{(t)}\right), a_{i}^{(t)} \in \mathbb{F}_{2^{16}}^{A}$ and $\left(b_{15}^{(t)}, b_{14}^{(t)}, \ldots, b_{1}^{(t)}, b_{0}^{(t)}\right), b_{i}^{(t)} \in \mathbb{F}_{{ }^{16}}^{B}$ respectively for LFSR-A and LFSR-B. Referring to Figure 1, the elements $a_{0}^{(t)}$ and $b_{0}^{(t)}$ are the elements to first exit the LFSRs. The LFSRs 
produce sequences $a^{(t)}$ and $b^{(t)}, t \geq 0$ which are given by the expressions

$$
a^{(t+16)}=b^{(t)}+\alpha a^{(t)}+a^{(t+1)}+\alpha^{-1} a^{(t+8)} \bmod g^{A}(\alpha)
$$

and

$$
b^{(t+16)}=a^{(t)}+\beta b^{(t)}+b^{(t+3)}+\beta^{-1} b^{(t+8)} \bmod g^{B}(\beta),
$$

where the initial states of the LFSRs are given by $\left(a^{(15)}, a^{(14)}, \ldots, a^{(0)}\right)$ and $\left(b^{(15)}, b^{(14)}, \ldots\right.$, $\left.b^{(0)}\right)$. We would like to emphasize the notation here; $a^{(t)}$ means the symbol produced by the linear recursion in Equation 3 at time $t$, whereas $a_{i}^{(t)}, 0 \leq i \leq 15$ are the values of the cells in the LFSR-A at time $t$. In the case of $\alpha$ and $\beta$, the notation $\alpha^{-1}$ and $\beta^{-1}$ are the inverses in the respective implemented fields.

As the reader might notice, we are a bit sloppy in Equation 3 and Equation 4 and apply the field addition operation between elements of different fields, but it should be interpreted as an implicit bit pattern preserving conversion between the fields.

Each time we update the LFSR part, we clock LFSR-A and LFSR-B 8 times, i.e., 256 bits of the total 512-bit state will be updated in a single step, and the two taps $T 1$ and $T 2$ will have fresh values. In Appendix A we give the proof that this circular construction gives the maximum cycle length of $2^{512}-1$.

The tap $T 1$ is formed by considering $\left(b_{15}, b_{14}, \ldots, b_{8}\right)$ as a 128 -bit word where $b_{8}$ is the least significant part. Similarly, $T 2$ is formed by considering $\left(a_{7}, a_{6}, \ldots, a_{0}\right)$ as a 128-bit word where $a_{0}$ is the least significant part. The mapping is pictured in Figure 2, and the expressions are given by

$$
\begin{aligned}
& T 1^{(t)}=\left(b_{15}^{(8 t)}, b_{14}^{(8 t)}, \ldots, b_{8}^{(8 t)}\right), \\
& T 2^{(t)}=\left(a_{7}^{(8 t)}, a_{6}^{(8 t)}, \ldots, a_{0}^{(8 t)}\right) .
\end{aligned}
$$

LFSR-A

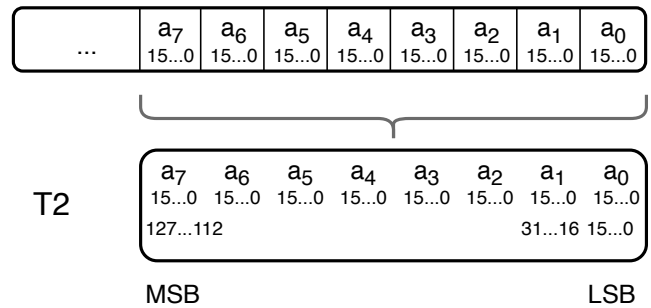

LFSR-B

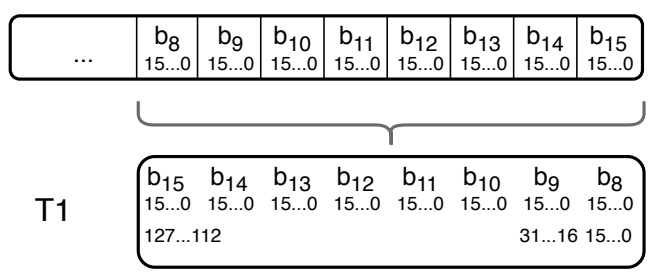

MSB

LSB

Figure 2: Mapping the 16-bit words of the LFSRs into 128-bit words $T 1$ and T2.

We will now turn to the FSM. The FSM takes the two blocks $T 1$ and $T 2$ from the LFSR part as inputs and produces a 128-bit keystream as output. $R 1, R 2$, and $R 3$ are 128-bit registers, $\oplus$ denotes a bitwise XOR operation, and $\boxplus_{32}$ denotes a parallel application of four additions modulo $2^{32}$ over each sub-word. So the four 32-bit parts of the 128-bit words are added with carry, but the carry does not propagate from a lower 32-bit word to the higher. 
The output, $z^{(t)}$ at time $t \geq 0$, is given by the expression

$$
z^{(t)}=\left(R 1^{(t)} \boxplus_{32} T 1^{(t)}\right) \oplus R 2^{(t)} .
$$

Registers $R 2$ and $R 3$ are updated through a full AES encryption round function as shown in Figure 3, see [oST01] for details. Let us denote the AES encryption round function by

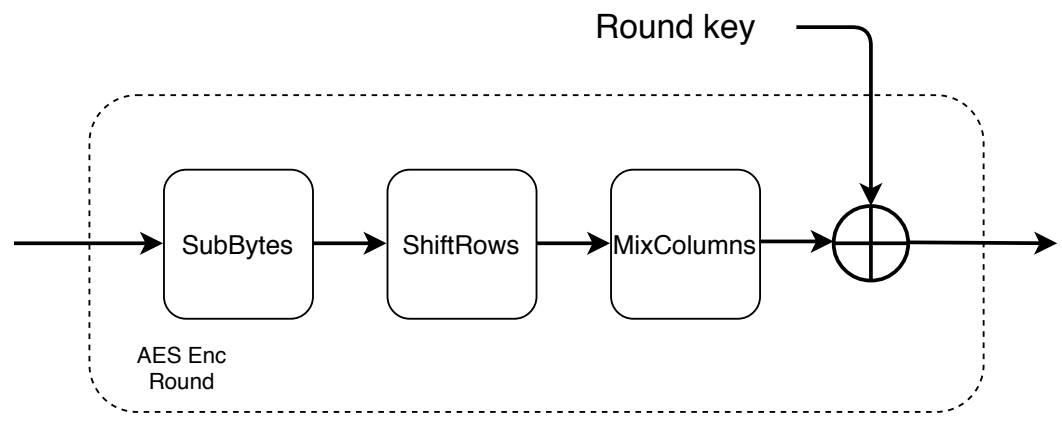

Figure 3: Internal functions of the AES encryption round function.

$A E S^{R}(I N, K E Y)$. The mapping between the 128-bit registers and the state array of the AES round function follows the definition in [oST01], and is pictured in Figure 4.

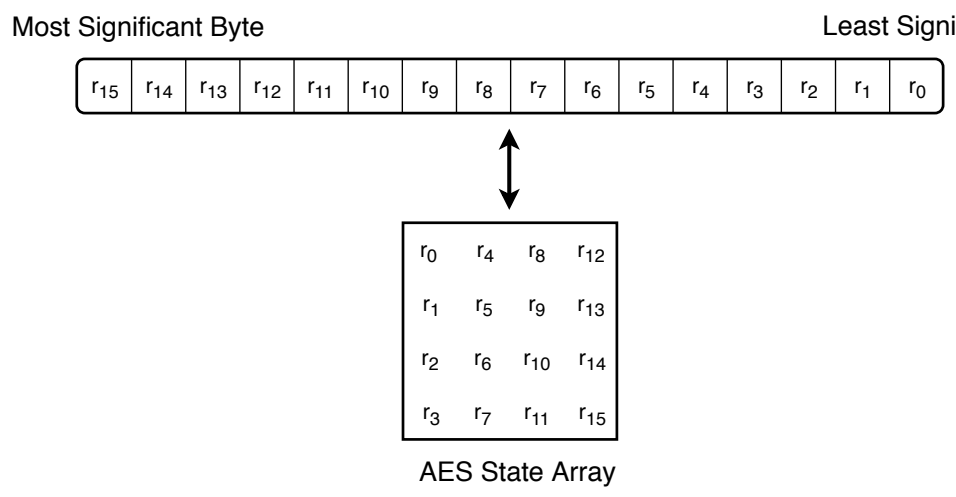

Figure 4: Mapping between a 128-bit register value and the state array of the AES round function.

We can now write the update expressions for the registers as

$$
\begin{aligned}
& R 1^{(t+1)}=\sigma\left(R 2^{(t)} \boxplus_{32}\left(R 3^{(t)} \oplus T 2^{(t)}\right)\right), \\
& R 2^{(t+1)}=A E S^{R}\left(R 1^{(t)}, C 1\right), \\
& R 3^{(t+1)}=A E S^{R}\left(R 2^{(t)}, C 2\right) .
\end{aligned}
$$

The values of the two round key constants $C 1$ and $C 2$ are set to zero, and $\sigma$ is a byte-oriented permutation given by

$$
\sigma=[0,4,8,12,1,5,9,13,2,6,10,14,3,7,11,15] .
$$

This should be interpreted as byte 0 is moved to position 0 , byte 4 is moved to position 1 , byte 8 is moved to position 2 , and so on. Position 0 is the least significant byte in accordance to the mapping described above. The chosen $\sigma$ implements the transposition of the mapped AES state matrix. 


\subsection{Initialization}

Initialization is done as described in this subsection. The algorithm has a 256-bit key $K$ and a 128-bit initialization vector(IV) as inputs. The key is denoted by

$$
K=\left(k_{15}, k_{14}, \ldots, k_{1}, k_{0}\right)
$$

where each $k_{i}, 0 \leq i \leq 15$, is a 16 -bit vector. The IV vector is denoted by

$$
I V=\left(i v_{7}, i v_{6}, \ldots, i v_{1}, i v_{0}\right)
$$

where again each $i v_{i}, 0 \leq i \leq 7$, is a 16 -bit vector.

The first step of the initialization is to load the key and IV into the LFSRs by assigning

$$
\left(a_{15}, a_{14}, \ldots, a_{0}\right)=\left(k_{7}, k_{6}, \ldots, k_{0}, i v_{7}, i v_{6}, \ldots, i v_{0}\right)
$$

and

$$
\left(b_{15}, b_{14}, \ldots, b_{0}\right)=\left(k_{15}, k_{14}, \ldots, k_{8}, 0,0, \ldots, 0\right) .
$$

Note that $\left(b_{7}, \ldots, b_{0}\right)$ will have a non-zero value when SNOW-V is used in AEAD-mode, see Section 4.

Then the initialization consists of 16 steps where the cipher is updated in the same way as in the running-key mode, with the exception that the 128-bit output $z$ is not an output but is xored into the LFSR structure to positions $\left(a_{15}, a_{14}, \ldots, a_{8}\right)$ in every step. Additionally, at the two last steps of the initialization phase, we xor the key into the $R 1$ register, inspired by [HK18]. We also limit the keystream length to a maximum of $2^{64}$ for a single pair of key and IV vectors, and each key may be used with a maximum of $2^{64}$ different IV vectors.

The pseudocode in Algorithm 1 clarifies the procedure.

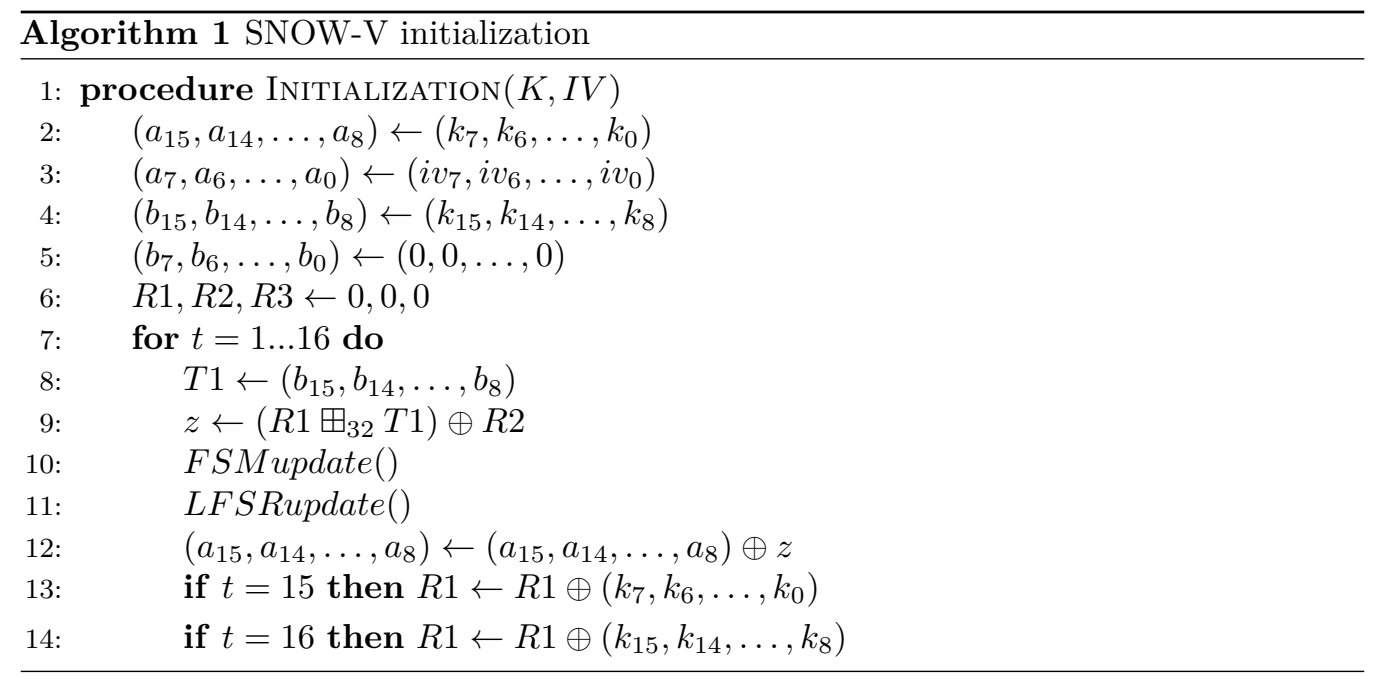

This completes the description of SNOW-V, and the full algorithm can be summarized in the pseudocode as in Algorithm 2, Algorithm 3, and Algorithm 4.

\section{Security analysis}

The main and most important design criterion is the security of the design. This section contains a brief analysis for a number of possible standard attack approaches. Before 

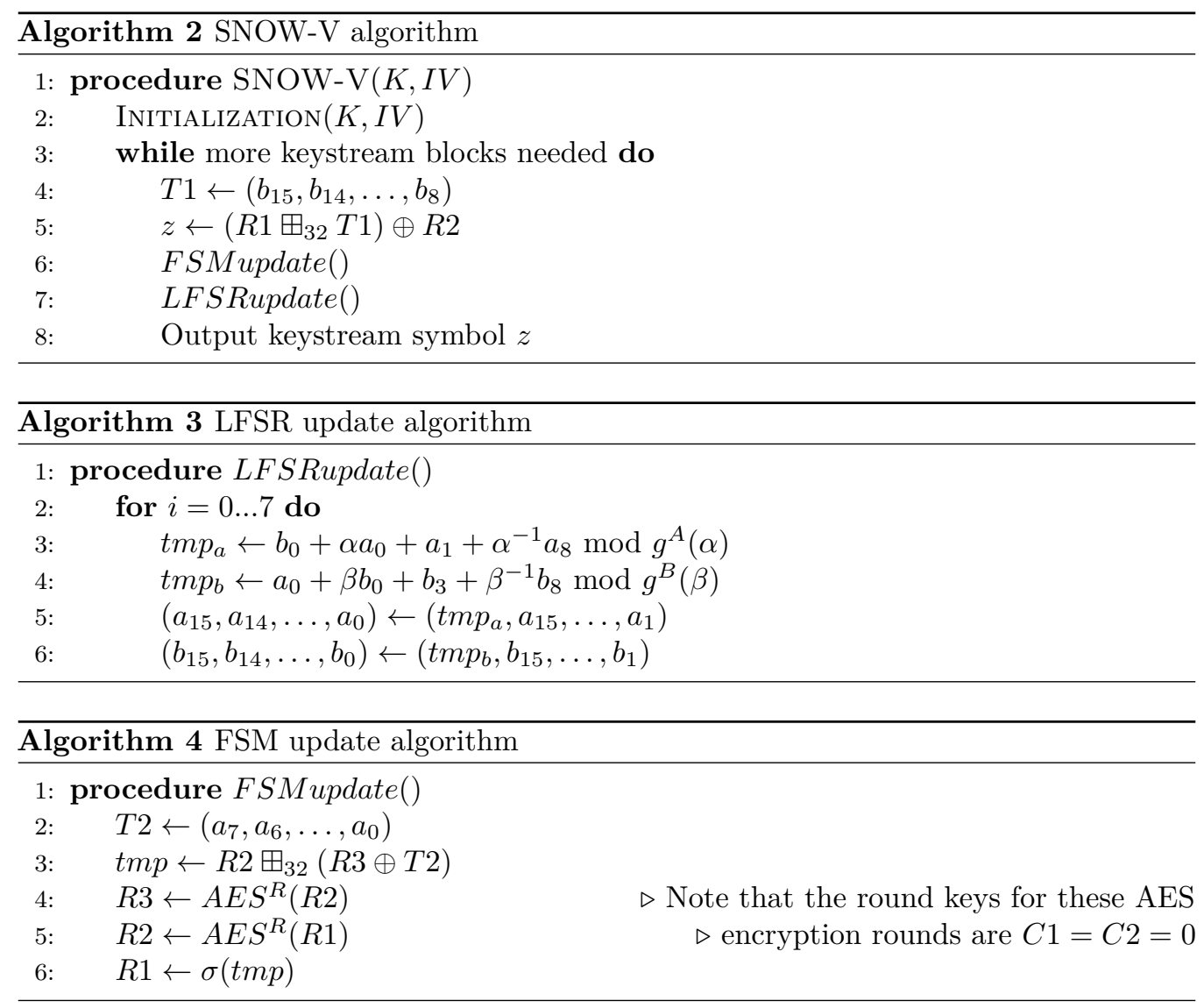

going into the details of various attacks, we need to have a clear picture of the expected security. We have the target of providing 256-bit security in SNOW-V, by which we mean that we claim that the total cost of finding the secret key given some keystreams is not significantly smaller than $2^{256}$ simple operations.

The use of the algorithm is limited to keystreams of length at most $2^{64}$ and we also limit the number of different keystreams that are produced for a fixed key to be at most $2^{64}$. There seem to be no use cases where it makes sense to violate this limitation. Although attacks beyond these limits are certainly of academic interest, an attack claiming to break the cipher should meet this requirement. In Subsection 3.1 and Subsection 3.4 we give some cryptanalysis results also for the case when $\sigma$ is replaced by the identity mapping $\sigma_{0}$. This may aid the understanding of the strength of different methods of cryptanalysis.

We also frequently compare with AES-256 in the GCM mode. We note that exhaustive key search of AES-256 requires computational cost around $2^{256}$. However, if used in the GCM mode, it actually takes complexity (and data) around $2^{64}$ to distinguish such keystreams from random. For SNOW-V, we claim that the security is never worse than the security of AES-256 in the GCM mode, for any kind of attack on the algorithmic level.

\subsection{Initialization attacks through MDM/AIDA/cube attacks}

Stream ciphers always have an initialization phase before producing keystream bits, during which the key and IV are loaded and mixed by running the cipher a few rounds (16 for SNOW-V) without giving outputs until the state becomes random-like. It should be difficult for a cryptanalyst to predict the keystream or get some information about the initial key according to the output after initialization. It then becomes vital to make sure 
that the key/IV loading has no fatal flaws and the initialization round is carefully chosen in order not to result in a resource waste (too many rounds) or some weakness (too few rounds).

A chosen IV attack is one type of attacks targeting this problem [Mj06, EJT07], in which the adversary attempts to build a distinguishing attack to introduce randomness failures in the output by selecting and running through certain IV values. The rationales behind are that: 1) a stream cipher can be regarded as a succession of single-valued boolean functions $f_{i}$ with each keystream bit as the output and key/IV as the inputs, and 2) any monomial coefficient in the algebraic normal form (ANF) representations of these Boolean functions should appear to be 1 (or 0 ) with probability $1 / 2$ if $f_{i}$ is drawn uniformly at random [Sta13]. If one can distinguish the output from a random distribution under some key/IV settings with acceptable complexity, the cipher is believed to be unsafe. In this attack, the adversary fixes the key and a subset of IV bits and runs through all possible values of the non-fixed IV bits, which are called a cube. The truth tables of the boolean functions can be derived, which are further used to compute the monomial coefficients of the ANF and compared with expected values. If there exists a big gap between them, the output is believed to be non-random. The best and most commonly used monomial is the maximum degree monomial (MDM) and the corresponding test is called MDM test. In [Sta10] one even allows setting arbitrary key values to build a non-randomness detector to further check whether the initialization is robust enough. It should be noted that the MDM test and AIDA (algebraic IV differential attack)/cube distinguishers [Vie07, DS09] are various forms of using higher order differentials [Lai94] on stream ciphers.

We employ the greedy MDM test algorithm in [Sta10] to test the SNOW-V initialization. We start with the worst 3-bit set under which the randomness result deviates the most from the expected value and gradually increase to a 24-bit set. Every time when one more bit is added from the remaining bits, we select the bit resulting in the worst randomness result until we get a 24-bit set (larger sets can be tested on more powerful computers). Figure 5 shows the maximum number of initialization rounds failing the MDM test under different bit set sizes for SNOW-V under permutation $\sigma$ and no permutation $\sigma_{0}$ (identity mapping). The results for 1, 2 and 3-bit sets are obtained through exhaustive search, while for the sets with larger sizes, the results are based on greedy searching from the obtained worst 3-bit set. It can be seen that the performance under $\sigma$ is better, indicating the cipher is mixed better and faster in this case. In both cases, roughly the first 7 rounds fail the MDM test, ensuring a large security gap to 16 rounds. One can also note that the number of rounds the MDM test can detect grows very slowly with the size of the set of key/IV bits that are exhausted. In an attack, one could consider sets of sizes up to 64 bits. This indicates that the 16 initialization rounds in SNOW-V should be enough for the cipher and that the output of the cipher has become random-like after the initialization. It also indicates that significantly reducing the number of rounds might be dangerous.

Recently, cube attacks based on division property have got a lot of research and application on multiple stream ciphers. Division property, proposed by Todo et al. in [Tod15], is a generalization of integral property being used to find integral distinguishers or launch cube attacks. Unlike traditional experimental attacks where the ciphers are regarded as black boxes, division property based attacks explore the internal structures of ciphers and trails of division property according to propagation rules for different operations. These rules could be expressed with some (in)equalities and the attacks are modeled as MILP(Mixed Integer Linear Programming) problems with certain constrains and objective functions. Some optimization tools such as Gurobi, Cplex could then help to solve the problems very efficiently and identify if the attacks are feasible or not in certain cases.

In [TIHM17], division property was introduced into cube attacks to evaluate the set of key bits $J$ involved in the superpoly given a certain cube $I$. After obtaining the set 


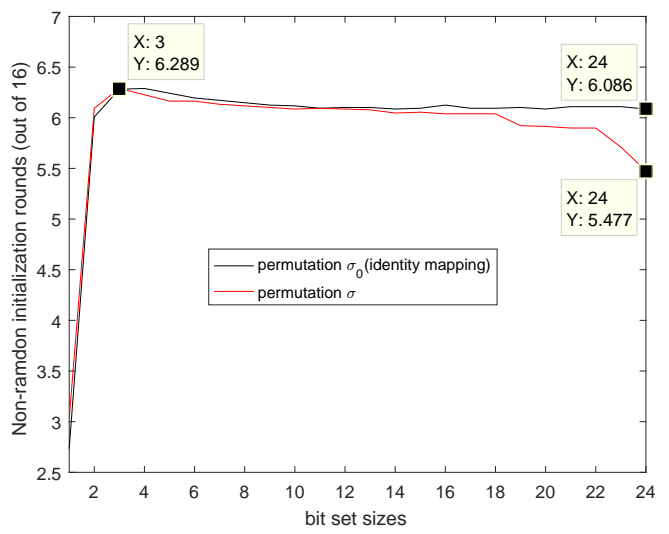

Figure 5: The maximum number of initialization rounds failing the MDM test under different bit set sizes.

$J$, attackers are able to recover the superpoly by building the truth table and further to recover part of the key by querying the encryption oracle. The time complexity of recovering the superpoly is $2^{|I|+|J|}$, which is feasible when $|I|+|J|$ is smaller than the security bit level. Authors in $\left[\mathrm{WHT}^{+} 18\right]$ further improved the attack by exploiting various algebraic properties of superpolies. They introduced a technique to evaluate the upper bound of the algebraic degree, denoted as $d$, of the superpoly to avoid recovering the coefficients of monomials with degrees larger than $d$. Hence, only $\left(\begin{array}{c}|J| \\ \leq d\end{array}\right)$ coefficients need to be recovered and the time complexity reduces to $2^{|I|} \times\left(\begin{array}{c}|J| \\ \leq d\end{array}\right)$.

We evaluate SNOW-V with division property based cube attacks using the method described above. The MILP model of division property for SNOW-V which can evaluate all division trails when the initialization rounds are reduced to $R$ is illustrated in Algorithm 5 . We first load key and IV bits to the LFSR state and initialize $R 1, R 2, R 3$ to 0 according to the initialization specification of SNOW-V (Algorithm 1). Since $K$ and $I V$ are loaded into LFSR states through one-by-one mapping, we do not introduce more intermediate variables in the model. In every round, we deal with the first and last 7 iterations of LFSR update differently since they have different propagation trails: the function LFSRupdate for the last 7 LFSR updates consists of copy, xor, multiplication (with $\alpha, \beta, \alpha^{-1}, \beta^{-1}$ ), shift to update the LFSR state while for the first round, the function LFSRupdateFirstIter involves in more complicated copy trails to get $T 1$ and $T 2$. The funcAES function is four parallel AES rounds consisting of sbox, shiftrow and mixcolumn whose propagation rules could be found in [Tod15]. The function funcModAdd consists of 4 parallel modular additions whose propagation rule has been established in [SWW17] and funcXor represents xor rule for 128 bits. The functions of multiplication and mixcolumn have consistent characteristic: the sum of input equals to the sum of output in terms of division property while the functions shift, shiftrow and sigma only permute the division property vectors.

Table 2: Cube attacks on reduced-rounds of SNOW-V (values in round brackets are for the case with $\sigma$ as identity $\left(\sigma_{0}\right)$ where the value is different).

\begin{tabular}{|c|c|c|c|c|c|}
\hline Rounds & 3 & 4 & 5 & 6 & 7 \\
\hline cube size $|I|$ & 15 & 40 & 128 & 128 & 128 \\
degree $d$ & 17 & $145(27)$ & $256(106)$ & $256(254)$ & 256 \\
involved key size $|J|$ & $131(113)$ & $256(96)$ & 256 & 256 & 256 \\
time complexity & $2^{84.9}\left(2^{81.1}\right)$ & $>2^{256}\left(2^{119.5}\right)$ & $>2^{256}$ & $>2^{256}$ & $>2^{256}$ \\
\hline
\end{tabular}




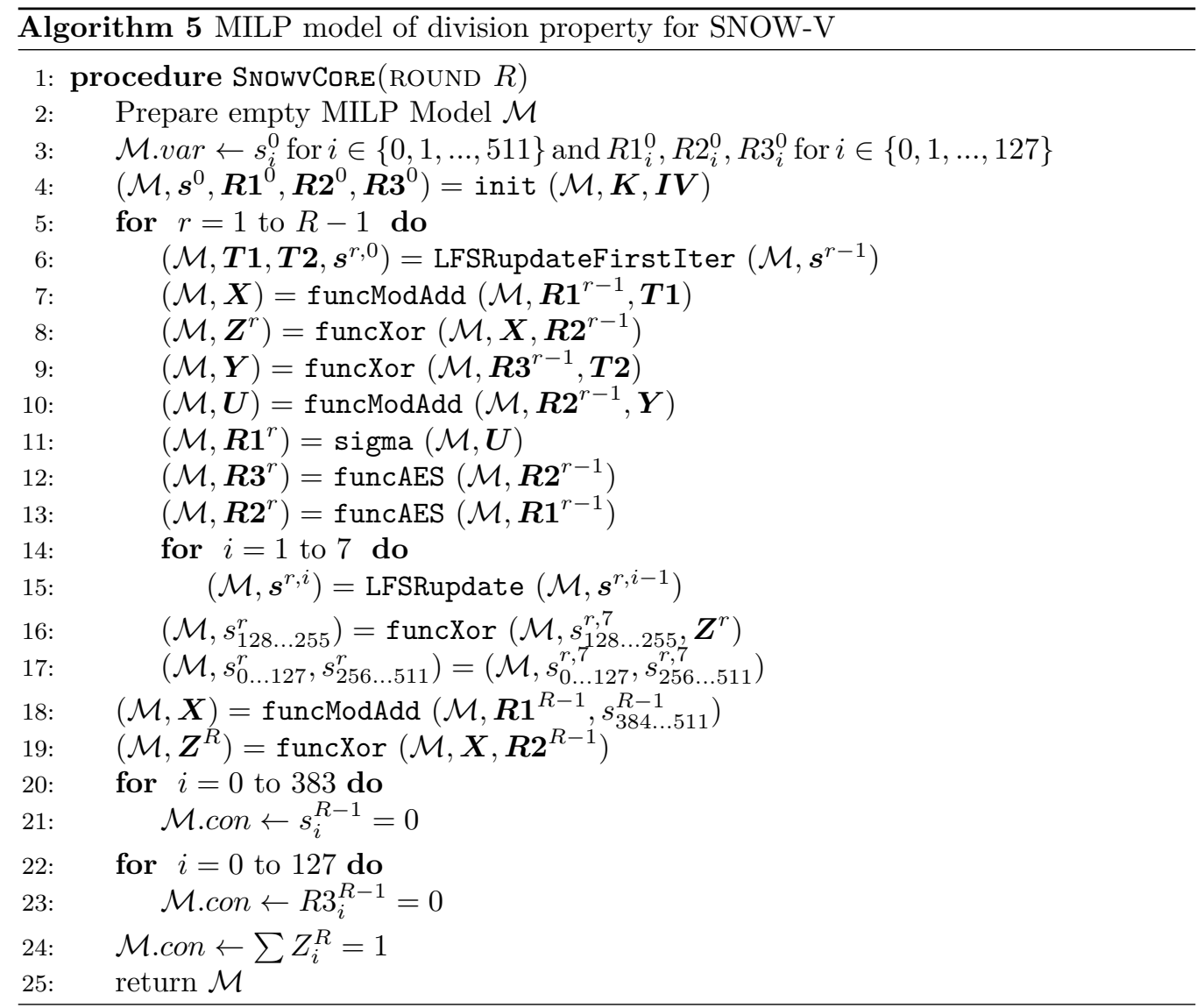

We tried different cubes and Table 2 listed some examples under which adversaries have good advantages for permutations $\sigma$ and $\sigma_{0}$. The time complexity in the table shows the time complexity of superpoly recovery. One can see, all key bits are involved in the superpolies from the 4-th round under permutation $\sigma$ while 5 -th round under $\sigma_{0}$, which indicates the good mixing effect after 4(or 5 ) rounds and guarantees a large enough security margin for 16 rounds. The results match well with the research on division property based distinguishing attacks on AES in [Tod15], where only 4-round distinguisher could be found with $2^{120}$ plaintexts and the conclusion in [SWW16] that integral distinguishers for AES based on division property covering more than four rounds probably do not exist.

\subsection{Other initialization attacks}

Another attack possibility is to launch a differential attack, either in the IV bits only, or in combination with key bits. The latter would then lead to a related-key attack. Since the initialization contains 16 rounds, each including two applications of the AES encryption round function, the differential would have to go through a lot of highly nonlinear operations, which makes this approach less successful.

Finally, a further option is the slide attacks [BW99]. Such sliding properties have been considered on previous versions in the SNOW family [KY11]. The idea is to have the same initial state for two different key/IV pairs in different time instances. Then they will produce the same keystream with the difference of a shift in time. Since the required IV values vary with the choice of key bits, it is questionable whether such an approach is useful at all in cryptanalysis, but at least it indicates that the cipher is not to be considered as a random function of both the key and IV. For SNOW-V such properties would still be 
much more difficult to find, due to the update of 128-bit blocks in each time instance and the use of the FP(1)-mode [HK18] in the initialization.

\subsection{Time/Memory/Data tradeoff attacks}

A Time/Memory/Data tradeoff (TMD-TO) attack is a generic method of inverting ciphers by balancing between spent time, required memory and obtained data, which can be much more efficient and applicable than an exhaustive key search attack. Some stream ciphers are vulnerable to TMD-TO attacks, and their effective key lengths (e.g., $n$-bit) could then be reduced towards the birthday bound (i.e., $n / 2$ ), typically happening if the state size is small. A well known such attack on A5/1 was given in [BSW01].

The TMD-TO attacks have two phases: a preprocessing phase, during which the mapping table from different secret keys or internal states to keystreams is computed and stored with time complexity $P$ and memory $M$; and a real-time phase, when attackers have intercepted $D$ keystreams and search them in the table with time complexity $T$, expecting to get some matches and further recover the corresponding input. By balancing between parameters $P, D, M$, and $T$ under some tradeoff curves, attackers can launch attacks according to their available time, memory and data resources. The most popular tradeoffs are Babbage-Golic (BG) [Bab95, Gol97] and Biryukov-Shamir (BS) [BS00] tradeoff with curves $T M=N, P=M$ with $T \leq D$; and $T M^{2} D^{2}=N^{2}, P=N / D$ with $T \geq D^{2}$, where $N$ is the input space, respectively. Attackers can try to reconstruct the internal state at a specific time or recover the secret key.

The rationale behind the TMD-TO attacks that try to reconstruct the internal state is that in many stream ciphers, the internal state update process is invertible, which means that if an attacker manages to reconstruct an internal state at any specific time, it can not only obtain subsequently generated keystreams by running the cipher forwards, but also recover previous states iteratively and further get the underlying secret key by running backwards. But for the SNOW-V case, attackers have no obvious ways to reconstruct the internal state, since SNOW-V has a large internal state with 896 bits $(2 \times 256$-bit LFSRs $+3 \times 128$-bit registers), which is 3.5 times the secret key length. The best attack complexity achieved is under BG tradeoff with point $T=M=D=N^{1 / 2}=2^{448}$, which is still much worse than the exhaustive key search attack. Actually, SNOW-V satisfies the rule derived from TMD-TO attacks in [Gol97] and widely applied in the design of new ciphers, that the size of the internal state should be at least twice the size of the secret key to get the expected security level.

Moreover, in SNOW-V, attackers would get even less even if they reconstructed an internal state. While computing subsequent keystreams corresponding to that specific IV is still possible, they can not trivially recover the secret key or keystreams under other IV values. This is due to the key masking to the register $R 1$ at the last two rounds of initialization, which represents an instantiation of the $F P(1)$-mode introduced in [HK18].

Attackers can also try to recover the secret key directly. To do so, some mappings from different key/IV pairs to generated keystream segments are firstly pre-computed and stored [HS05, DK08]. If attackers get some keystream data under different secret keys corresponding to these IV values, they can search them in the table to expect a collision and further recover some of the secret keys directly. The tradeoff curves are still the same as that to recover the internal states except $N$ is now changed to be the size of the set of all possible $(K, I V)$ pairs. In the SNOW-V case, the sizes of key and IV spaces are $2^{256}$ and $2^{128}$, respectively. Two typical points for BG and BS attacks are $T=D=M=2^{192}$ and $T=2^{256}, D=M=2^{128}$. Someone would question that the efficient size of the key in the first tradeoff is reduced from 256 to 192 bits, but actually, no ciphers including AES-256 can be immune to this as long as their IV sizes are smaller than the key sizes. In any case, the corresponding multikey attacks on AES-256 are not more costly. 


\subsection{Linear distinguishing attacks and correlation attacks}

Traditionally, the main threat against stream ciphers has been various types of linear attacks, either in the form of distinguishing attacks on the keystreams, or state recovery attacks through correlation attacks. The basic foundations of correlation attacks can be found in papers like [CJS01, CJM02] and an overview of distinguishing attacks is to be found in [HJB09].

The basic technique for these types of attacks is to use linear approximations of the nonlinear operations used in the cipher and then derive a linear relationship between output values from different time instances. Such a relationship will then hold only as a very rough approximation, which in turn can be thought of as a linear function of some given output bits being considered as a sample drawn from a nonuniform distribution. This approach may give a distinguishing property for the keystream. If the relationship also involves state bits, the same arguments may give samples that are highly noisy observations of state bits, which in turn may be linear combinations of the original initial state. This may give a way to recover the state and that is the foundation of a correlation attack.

For SNOW 2.0, several distinguishing attacks and correlation attacks have been proposed [NW06, ZXM15]. The basic idea has been to approximate the FSM part through linear masking and then to cancel out the contributions of the registers by combining expressions for several keystream words. We should note that this kind of attacks tend to require an extremely large length of the keystream. Also, no significant attack of this type on SNOW $3 \mathrm{G}$ has been published. We now consider a similar approach for making some basic arguments on SNOW-V.

We recall the FSM equations:

$$
\begin{aligned}
z^{(t)} & =\left(R 1^{(t)} \boxplus_{32} T 1^{(t)}\right) \oplus R 2^{(t)}, \\
R 1^{(t+1)} & =\sigma\left(R 2^{(t)} \boxplus_{32}\left(R 3^{(t)} \oplus T 2^{(t)}\right)\right), \\
R 2^{(t+1)} & =A E S^{R}\left(R 1^{(t)}\right), \\
R 3^{(t+1)} & =A E S^{R}\left(R 2^{(t)}\right) .
\end{aligned}
$$

A linear approximation of the FSM would then try to cancel out the contribution from the registers, leaving keystream symbols and the LFSR contribution. Assume that the value of the registers at some time $t$ is $(\hat{R} 1, \hat{R} 2, \hat{R} 3)$. Then we have

$$
\begin{aligned}
z^{(t)} & =\left(\hat{R} 1 \boxplus_{32} T 1^{(t)}\right) \oplus \hat{R} 2, \\
R 1^{(t+1)} & =\sigma\left(\hat{R} 2 \boxplus_{32}\left(\hat{R} 3 \oplus T 2^{(t)}\right)\right), \\
R 2^{(t+1)} & =A E S^{R}(\hat{R} 1), \\
R 3^{(t+1)} & =A E S^{R}(\hat{R} 2) .
\end{aligned}
$$

For time $t+1$,

$$
z^{(t+1)}=\left(\sigma\left(\hat{R} 2 \boxplus_{32}\left(\hat{R} 3 \oplus T 2^{(t)}\right)\right) \boxplus_{32} T 1^{(t+1)}\right) \oplus A E S^{R}(\hat{R} 1) .
$$

It is now straight-forward to see that since $z^{(t)}$ depends only on $\hat{R} 1$ and $z^{(t+1)}$ depends on both $\hat{R} 1$ and $\hat{R} 2$, there can be no biased linear approximation using only $z^{(t)}$ and $z^{(t+1)}$. So the minimum number of equations that needs to be considered is three. To simplify coming derivations, we introduce the expressions for $z^{(t-1)}$, i.e.,

$$
z^{(t-1)}=\left(\left(A E S^{R}\right)^{-1}(\hat{R} 2) \boxplus_{32} T 1^{(t-1)}\right) \oplus\left(A E S^{R}\right)^{-1}(\hat{R} 3),
$$

and seek a biased expression involving $z^{(t-1)}, z^{(t)}, z^{(t+1)}$. 
Consider all 128-bit variables as column vectors of 16 bytes. Then $A E S^{R}(X)$ can be written as $L \cdot S(X)$, where $S$ is an application of the 16 AES S-Boxes, one on each byte of $X$, and $L$ is a linear transformation over the 16 bytes (including both ShiftRow and MixColumn). Furthermore, $\left(A E S^{R}\right)^{-1}(X)=S^{-1}\left(L^{-1} \cdot X\right)$. We now introduce simplifications to the SNOW-V algorithm to show the best results on versions weaker than the proposed algorithm.

\subsubsection{Analysis of the bias using $\sigma_{0}$ and using byte-wise addition $\boxplus_{8}$}

We assume that there is no byte-wise permutation, i.e., $\sigma_{0}$ is just the identity. Furthermore, instead of $\boxplus_{32}$ we consider a modulo addition which is restricted to each byte, denoted $\boxplus_{8}$. With this simplification all operations are byte-oriented and the investigation of linear approximations is easier.

Let us now seek a byte-oriented linear approximation. We examine the following three equations.

$$
\begin{aligned}
z^{(t-1)} & =\left(S^{-1}\left(L^{-1} \cdot \hat{R} 2\right) \boxplus_{8} T 1^{(t-1)}\right) \oplus S^{-1}\left(L^{-1} \cdot \hat{R} 3\right), \\
z^{(t)} & =\left(\hat{R} 1 \boxplus_{8} T 1^{(t)}\right) \oplus \hat{R} 2, \\
L^{-1} z^{(t+1)} & =L^{-1}\left(\left(\hat{R} 2 \boxplus_{8}\left(\hat{R} 3 \oplus T 2^{(t)}\right)\right) \boxplus_{8} T 1^{(t+1)}\right) \oplus S(\hat{R} 1) .
\end{aligned}
$$

Let $X_{i}$ denote the $i$ th byte of a vector of bytes $X$. Summing up the three $z$-terms on the left side and taking byte 0 gives us

$\left[z^{(t-1)} \oplus z^{(t)} \oplus L^{-1} z^{(t+1)}\right]_{0}=\left[N 1 \oplus N 2 \oplus N 3 \oplus T 1^{(t)} \oplus T 1^{(t-1)} \oplus L^{-1}\left(T 2^{(t)} \oplus T 1^{(t+1)}\right)\right]_{0}$,

where

$$
\begin{aligned}
& N 1=\left(S^{-1}\left(L^{-1} \cdot \hat{R} 2\right) \boxplus_{8} T 1^{(t-1)}\right) \oplus S^{-1}\left(L^{-1} \cdot \hat{R} 2\right) \oplus T 1^{(t-1)}, \\
& N 2=\left(\hat{R} 1 \boxplus_{8} T 1^{(t)}\right) \oplus S(\hat{R} 1) \oplus T 1^{(t)}, \\
& N 3=\underbrace{L^{-1}\left(\left(\hat{R} 2 \boxplus_{8}\left(\hat{R} 3 \oplus T 2^{(t)}\right) \boxplus_{8} T 1^{(t+1)}\right) \oplus T 2^{(t)} \oplus T 1^{(t+1)}\right) \oplus \hat{R} 2}_{\text {Linear part } A} \\
& \oplus S^{-1}(\underbrace{L^{-1} \cdot \hat{R 2}}_{\text {Linear part } B}) \oplus S^{-1}(\underbrace{L^{-1} \cdot \hat{R 3}}_{\text {Linear part } C}) .
\end{aligned}
$$

The general idea is that $[N 1 \oplus N 2 \oplus N 3]_{0}$ is a biased distribution. It is true because the (types of) noise variables $n 1, n 2, n 3$ defined below are biased, which can be checked:

$$
\begin{aligned}
& n 1=\left(x \boxplus_{8} y\right) \oplus x \oplus y, \\
& n 2=\left(x \boxplus_{8} y\right) \oplus S(x) \oplus y, \\
& n 3=\left(x \boxplus_{8} y\right) \oplus x \oplus S(x) \oplus y .
\end{aligned}
$$

Each of the noise terms $N 1, N 2, N 3$ given above are of the above types and hence the sum of them can also be a biased distribution.

Computation of the bias: it remains to compute the bias and the $N 3_{0}$ part is the most complicated case (although we are computing the bias of $[N 1 \oplus N 2 \oplus N 3]_{0}$ as there is a dependence between $N 1$ and $N 3$ ). The noise $N 3$ at byte 0 can be rewritten as:

$$
N 3_{0}=\sum_{i=0}^{3}\left(c_{i} \cdot U_{i}\right) \oplus \hat{R} 2_{0} \oplus S^{-1}\left(\sum_{i=0}^{3}\left(c_{i} \cdot \hat{R} 2_{i}\right)\right) \oplus S^{-1}\left(\sum_{i=0}^{3}\left(c_{i} \cdot \hat{R} 3_{i}\right)\right),
$$

where the coefficients are $c=\left[\begin{array}{llll}e & b & d & 9\end{array}\right]$. First note that $U_{i}$ is the variable corresponding to $U_{i}=\left(\hat{R} 2_{i} \boxplus_{8}\left(\hat{R} 3_{i} \oplus T 2_{i}^{(t)}\right) \boxplus_{8} T 1_{i}^{(t+1)}\right) \oplus T 2_{i}^{(t)} \oplus T 1_{i}^{(t+1)}$, for $0 \leq i \leq 3$. Note also that 
$L^{-1}$ corresponds to first applying the inverse MixColumn and then the inverse ShiftRow (which does not change the position of byte 0 ).

The idea of computing the distribution is now simple. We assume that we have 8-bit adders $\boxplus_{8}$ instead of the original 32-bit ones, keeping all operations within bytes. We try all combinations of the first bytes of the inputs $\hat{R} 2_{0}, \hat{R} 3_{0}, T 2_{0}^{(t)}, T 1_{0}^{(t+1)}$ which leads to a partial sum as a 3-byte value denoted $A|B| C$, as marked in the equations above. Thus, we can compute the distribution $D_{0}(A|B| C)$. We also compute similar distributions for every "slice" of the inputs, $D_{k}(A|B| C), k=0,1,2,3$, corresponding to inputs $\hat{R} 2_{k}, \hat{R} 3_{k}, T 2_{k}^{(t)}, T 1_{k}^{(t+1)}$.

The XOR-convolution of the computed distributions of the partial linear expressions gives the combined distribution of the triple $A|B| C$ over all possible 32-bit inputs. Having that total distribution, it is then easy to construct the 8-bit distribution of $N 3_{0}$.

Let $D$ be a distribution of a noise variable $X$. Then we compute the bias denoted $\epsilon(X)=\epsilon(D)$ using the Squared Euclidean Imbalance as in [ZXM15], defined through

$$
\epsilon(D)=|D| \sum_{x=0}^{|D|-1}\left(D(x)-\frac{1}{|D|}\right)^{2} .
$$

The number of samples needed to distinguish a source of noise $D$ from random is roughly $O(1 / \epsilon)$. The results when we use 8-bit adders $\boxplus_{8}$, and $\sigma$ identity are as follows: $\epsilon(N 1)>$ $1, \epsilon(N 2) \approx 2^{-2.9}, \epsilon(N 3) \approx 2^{-46.0}$, and

$$
\epsilon\left(N_{t o t}\right) \approx 2^{-53.5}, \quad \epsilon\left(2 \times N_{t o t}\right) \approx 2^{-106.8}, \quad \epsilon\left(3 \times N_{t o t}\right) \approx 2^{-160.2}, \quad \epsilon\left(4 \times N_{t o t}\right) \approx 2^{-213.6} .
$$

Here the $N 1, N 2, N 3$ denotes the partial noise as described above and $N_{t o t}$ represents the full noise of a single approximation, i.e., $N_{t o t}=[N 1 \oplus N 2 \oplus N 3]_{0}$. Finally, $i \times N_{t o t}$ denotes the noise obtained by a sum of $i$ such independent noise terms.

\subsubsection{Analysis of the bias using $\sigma_{0}$ and using 32-bit $\boxplus_{32}$}

In order to deal with 32-bit adders we should actually compute partial noise distributions $D_{k}$ that also correspond to different values of input and output carries $(0,1$, or 2$)$ and then perform sums and convolutions over matching distribution tables. It is computationally more demanding but not unreachable and we have computed the biases also in this case.

The results when using 32-bit adders $\boxplus$, and $\sigma$ as identity are as follows: $\epsilon(N 1)>$ $1, \epsilon(N 2) \approx 2^{-2.9}, \epsilon(N 3) \approx 2^{-46.4}$, and

$$
\epsilon\left(N_{t o t}\right) \approx 2^{-58.7}, \quad \epsilon\left(2 \times N_{t o t}\right) \approx 2^{-118.4}, \quad \epsilon\left(3 \times N_{t o t}\right) \approx 2^{-177.8}, \quad \epsilon\left(4 \times N_{t o t}\right) \approx 2^{-237.1} .
$$

\subsubsection{Using the bias in a fast correlation attack}

A detected bias can be used in a distinguishing attack if one can find, say, a weight 3 or weight 4 multiple of the polynomial corresponding to the byte-oriented sequence $W(t)=\left[T 1^{(t)} \oplus T 1^{(t-1)} \oplus L^{-1}\left(T 2^{(t)} \oplus T 1^{(t+1)}\right)\right]_{0}$. Since the LFSR feedback is defined in $\mathbb{F}_{2^{16}}$, it can be rewritten in $\mathbb{F}_{2^{8}}$ and there will be a linear recursion relation for $W(t)$ over $\mathbb{F}_{2^{8}}$. The complexity of finding a weight 3 or weight 4 multiple with general methods is far more than that of exhaustive key search. Instead, a fast correlation attack looks more promising. In such an attack the LFSR state at some initial time is considered as a length 64 byte vector $\mathbf{v}=\left(v_{0}, v_{1}, \ldots v_{63}\right), v_{i} \in \mathbb{F}_{2^{8}}$ and every $W(t)$ is written as a linear combination of initial state bytes, i.e., $W(t)=\mathbf{w}_{\mathbf{t}} \cdot \mathbf{v}^{T}$, where the vector $\mathbf{w}_{\mathbf{t}}$ is computed through the recursion for $W(t)$. If we now look for pairs of vectors $\mathbf{w}_{\mathbf{t}}$ and $\mathbf{w}_{\mathbf{t}^{\prime}}$ such that $\mathbf{w}_{\mathbf{t}} \oplus \mathbf{w}_{\mathbf{t}^{\prime}}$ is zero in the last $d$ entries, we can form the sum

$$
\left[z^{(t-1)} \oplus z^{(t)} \oplus L^{-1} z^{(t+1)}\right]_{0} \oplus\left[z^{\left(t^{\prime}-1\right)} \oplus z^{\left(t^{\prime}\right)} \oplus L^{-1} z^{\left(t^{\prime}+1\right)}\right]_{0}
$$


which approximates $W(t) \oplus W\left(t^{\prime}\right)$ with a noise which is the sum of two noise variables of the form Ntot, which has a bias of $2^{-118.4}$. In the attack we guess $64-d$ byte entries of the initial state and then we need to find in the order of $2^{118}$ pairs of vectors that have the same last $d$ entries. Through a birthday argument, assuming we generate all such values up to $t_{0}$, we need to have $t_{0}^{2} \approx 2^{118+8 d}$. For example, if $d=36$ then we need to guess $28 \cdot 8=224$ bits and we need to generate output until time $t_{0}=2^{203}$. Following the complexity estimations of e.g. [ZXM15] we end up with a complexity slightly below exhaustive key search. This however requires a keystream of length $t_{0}=2^{203}$, which can be compared to the maximum keystream length allowed which is $2^{64}$. The attack also uses memory of size $2^{203}$ units, each storing the necessary information of one time unit. So the relevance of such attacks is indeed questionable.

\subsubsection{Analysis of the bias using $\sigma$ as proposed}

In this scenario, we use $\sigma$ as given in Section 2. This presumably makes the bias of linear approximations for the FSM to be much smaller, and we will sketch some ideas on how to find good linear approximations. Let us again return to the equations for a three word approximation, and use 8-bit adders $\boxplus_{8}$ in order to simplify derivations:

$$
\begin{aligned}
z^{(t-1)} & =\left(S^{-1}\left(L^{-1} \cdot \hat{R} 2\right) \boxplus_{8} T 1^{(t-1)}\right) \oplus S^{-1}\left(L^{-1} \cdot \hat{R} 3\right), \\
z^{(t)} & =\left(\hat{R} 1 \boxplus_{8} T 1^{(t)}\right) \oplus \hat{R} 2, \\
z^{(t+1)} & =\left(\sigma\left(\hat{R} 2 \boxplus_{8}\left(\hat{R} 3 \oplus T 2^{(t)}\right)\right) \boxplus_{8} T 1^{(t+1)}\right) \oplus L \cdot S(\hat{R} 1) .
\end{aligned}
$$

We consider a byte-oriented linear approximation with left side $\Lambda_{0} z^{(t-1)} \oplus \Lambda_{1} z^{(t)} \oplus \Lambda_{2} z^{(t+1)}$, where $\Lambda_{i}$ are length 16 row vectors of bytes viewed as elements in $\mathbb{F}_{2^{8}}$.

Recall now that if a particular byte is only present once as a linear term or in an S-box expression in the right side, then the approximation will be unbiased. However, if it appears at least twice in different types of expressions, it is likely to give a biased contribution.

The first observation we can do is that the bytes in $\hat{R} 1$ appear only in two different expressions: as the direct value $\hat{R} 1$ in the expression for $z^{(t)}$ and as $L \cdot S(\hat{R} 1)$ in the expression for $z^{(t+1)}$. In turn, this means that $\Lambda_{1} z^{(t)} \oplus \Lambda_{2} z^{(t+1)}$ depends on $\hat{R} 1$ through $\Lambda_{1} \hat{R} 1 \oplus \Lambda_{2} L \cdot S(\hat{R} 1)$. It is biased only if every byte present in $\Lambda_{1} \hat{R} 1$ is also present in $\Lambda_{2} L \cdot \hat{R} 1$ and we come to the conclusion that $\Lambda_{2}=\Lambda_{1} L^{-1}$, in its straightforward case.

Now we consider the contributions from $\hat{R} 2$ and $\hat{R} 3$. For $\hat{R} 2$ we have contributions $\Lambda_{0} S^{-1}\left(L^{-1} \cdot \hat{R} 2\right), \Lambda_{1} \hat{R 2}$ and $\Lambda_{1} L^{-1} \sigma\left(\hat{R} 2 \boxplus_{8} \ldots\right)$. When $\sigma_{0}$ was used, we could simply consider byte $0\left(\Lambda_{0}=\Lambda_{1}=(1,0, \ldots, 0)\right)$ because it involved four bytes (byte $\left.0-3\right)$ and they all appeared at least twice in the above expression. But for the actual $\sigma$, this is no longer possible since $L^{-1} \sigma(\hat{R} 2)$ includes different bytes in a position, compared to $L^{-1} \cdot \hat{R} 2$.

We have not been able to find better approximations than the ones that include all 16 bytes of $\hat{R} 2$ and $\hat{R} 3$, and 4 bytes of $\hat{R} 1$ in the approximation. One such example would be $\Lambda_{0}=\Lambda_{1}=(1,1,1,1,0,0,0,0,0,0,0,0,0,0,0,0)$, which would nearly correspond to the sum of 4 approximations of the previous kind with $\sigma_{0}$ (no-permutation).

In our best attempt to approximate the FSM of the proposed algorithm, we have got the total noise (with $\boxplus_{8}$ ) having the bias (more details can be found in Appendix B):

$$
\epsilon\left(N_{t o t}\right) \approx 2^{-214.8} \quad \text { and } \quad \epsilon\left(2 \times N_{t o t}\right) \approx 2^{-429.7} .
$$

\subsection{Algebraic attacks}

In an algebraic attack the attacker derives a number of nonlinear equations in either unknown key bits or unknown state bits and solves the system of equations. In general, the problem of solving a system of nonlinear equations is not known to be solvable in 
polynomial time (even for quadratic equations), but some special cases might be solved efficiently [CKPSO0].

For SNOW 2.0 there was a very interesting algebraic attack on a simplified version, given in [BG05]. However, due to the use of three FSM registers instead of two, applying such an approach on SNOW-V does not give such a nice quadratic system as in [BG05].

So for a general algebraic attack, we should either target the key or the state. For the latter, one would need to use equations from 7 keystream blocks to be able to solve for the $7 * 128$ bit internal state. That would involve nonlinearity from 11 AES encryption round functions and $13 \boxplus_{32}$ operations. Instead, targeting the key bits would require stepping through the equations of the 16 initialization rounds together with the equations of two keystream blocks. Both these approaches give systems of nonlinear equations that appear to be much more difficult to solve than corresponding equations for AES-256. This is due to the use of the $\boxplus_{32}$ operation.

\subsection{Guess-and-determine attacks}

In a guess-and-determine attack one guesses part of the state and from the keystream equations, determines the value of other parts of the state. The goal is to guess as few bits as possible and determine as many as possible through keystream equations. For the case of SNOW-V, the equation $z^{(t)}=\left(R 1^{(t)} \boxplus_{32} T 1^{(t)}\right) \oplus R 2^{(t)}$ involves three unknown values, each of size 128 bits. In order to determine some state bits, one then has to guess two of them, i.e. guessing 256 bits. Then looking at the equation for $z^{(t+1)}$, it would require the guess of one more 128 bit value. This indicates that a guess-and-determine attack would not be successful.

\subsection{Other attacks}

We have not made any specific design choices to explicitly support implementations that should protect against side-channel attacks and fault attacks. So such attacks, if relevant for an application, have to be considered when the algorithm is implemented. In particular, information leakage from the CPU in a software implementation must be carefully considered.

\section{AEAD mode of operation}

The GMAC integrity and authentication algorithm specified in [Dwo07] can easily be adopted to work with SNOW-V to define an AEAD mode of operation. We will use notations from [Dwo07] in the following. In GCM, an unspecified block cipher is used in counter mode to encrypt the plaintext. Additionally, the block cipher is used to produce the final authentication $\operatorname{tag} T$, and to derive the key $H$ used in the function $G H A S H_{H}$.

When using SNOW-V together with the $G H A S H_{H}$ algorithm, the key $H$ is the very first keystream output $z^{(0)}$. Then we use keystream output $z^{(1)}$ as the final masking for the tag, similarly to the encrypted value of $J_{0}$ in [Dwo07]. To encrypt the $n$ plaintext blocks, we use the keystream outputs $z^{(2)}, \ldots, z^{(n+1)}$, feeding the ciphertext blocks into GHASH . $_{\text {. }}$

SNOW-V works as described in Section 2 with a single change. During initialization of the LFSRs, we set the lower part of the LFSR-B to the following hex values:

$$
\left(b_{7}, b_{6}, \ldots, b_{0}\right)=(6 D 6 F, 6854,676 E, 694 A, 2064,6 B 45,7865,6 C 41) .
$$

The hex values are the UTF8 encoding of the names of the authors.

An overview of how SNOW-V is used together with the $G H A S H_{H}$ algorithm is shown in Figure 6. The padding of the Additional Authenticated Data (AAD) and how to 


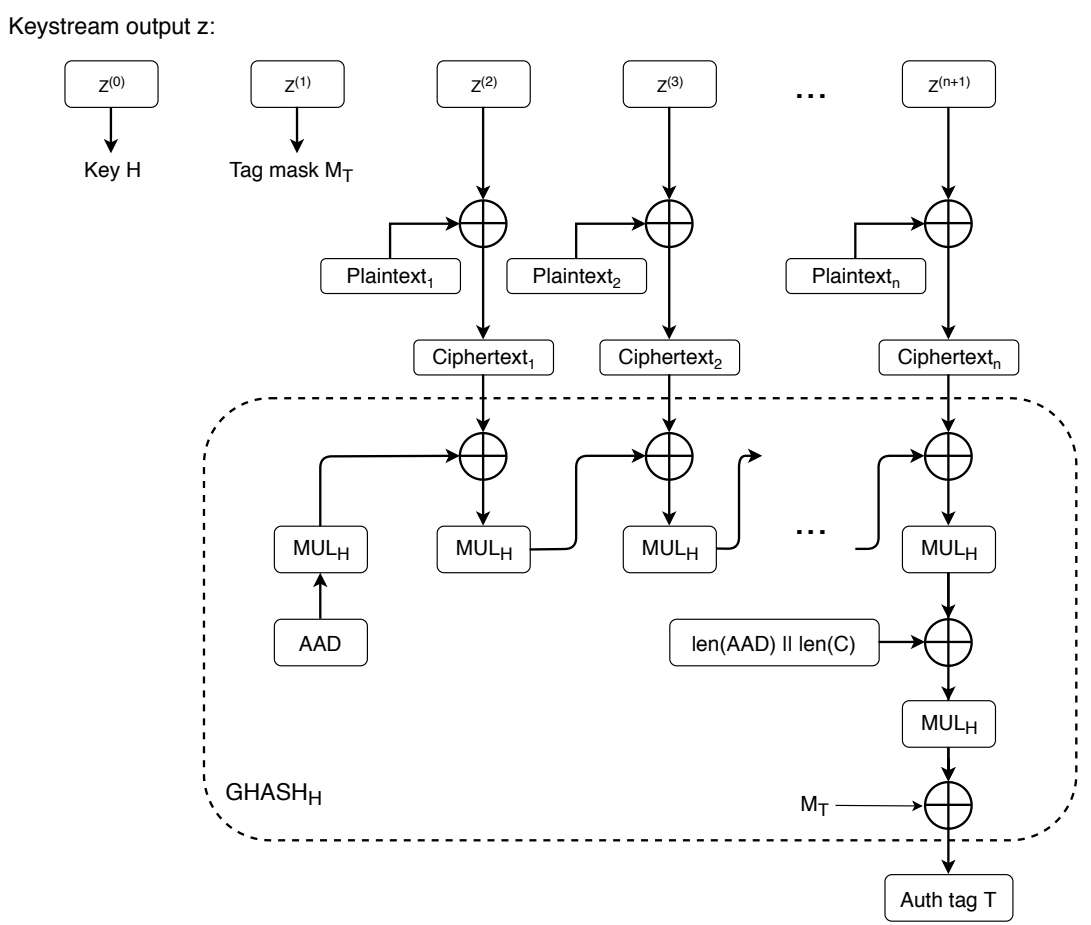

Figure 6: How SNOW-V is used together with $G H A S H_{H}$ to enable AEAD.

concatenate the length of the $\mathrm{AAD}$ and the length of the ciphertext $\mathrm{C}$ and all other restrictions on plaintext length and change of IV from [Dwo07] remain. We have only defined a new way to derive the counter mode keystream, and the additional key and tag mask needed in the GCM algorithm.

\section{Software implementation aspects}

One important change in future telecom networks is the virtualization of the network functions. This puts new requirements on the crypto algorithms used to protect the traffic in that it needs to execute fast in a pure software implementation on modern CPUs. According to [ITU17], the minimum requirements related to $5 \mathrm{G}$ radio interface are $10 \mathrm{Gbps}$ uplink and 20 Gbps downlink, at peak data rates. Classical encryption algorithms cannot reach these high speeds in pure software without any hardware support.

Nowadays, most of CPU vendors provide large registers and vectorized SIMD instructions, such as AVX2 set of instructions (intrinsics) that can execute over registers of up to 256 bits. Typical instructions include such functions as XOR, AND, nADD32, etc., applied to long registers, where, depending on the instruction, a single register can be represented as a vector of $8 / 16 / 32 / 64$-bit values.

AES is one of the most widely used crypto algorithms and it has received special support from CPU vendors in the form of SIMD instructions (AES-NI for Intel) that makes it possible to execute AES quite fast even on user-grade laptops. Crypto ciphers SNOW $3 \mathrm{G}$ and ZUC, standardized in $4 \mathrm{G}$, and other ciphers (to our best knowledge), cannot reach the speed even close to AES when AES-NI is used.

SNOW-V is designed to perform very fast in software, with the aim to utilize currently available SIMD instructions. However, even without AES-NI, SNOW-V can be implemented quite efficiently with $16 / 32 / 64$-bit registers. Our take-away is that if a given platform 
supports AES-NI then other SIMD instructions are also likely supported. If AES-NI is not available then AES-256 will be much slower than SNOW-V, and actually, slower than SNOW 3G as well. This section is written with Intel intrinsics notation, but similar implementations can likely be made on other CPUs, e.g. AMD and ARM. A comprehensive guide on Intel's intrinsics can be found in [Int18].

The FSM part of SNOW-V is quite straightforward to implement using 128-bit registers _-_m128i and AES-NI intrinsic function _mm_aesenc_si128(). For 4 parallel arithmetic additions one can use _mm_add_epi32( $)^{1}$. The 16 -byte permutation $\sigma$ can be done with _mm_shuffle_epi8().

The key to an efficient implementation of the LFSRs is choosing the right data structures. We propose to store the content of the two LFSRs in two 256-bit registers _-_m256i hi, lo, such that:

$$
\begin{aligned}
\text { lo }[127 . .0 \text { bits }] & =\left\{a_{7}, \ldots, a_{0}\right\} & \text { hi }[127 \ldots 0 \text { bits }] & =\left\{a_{15}, \ldots, a_{8}\right\} \\
\text { lo }[255 \ldots 128 \text { bits }] & =\left\{b_{7}, \ldots, b_{0}\right\} & \text { hi }[255 \ldots 128 \text { bits }] & =\left\{b_{15}, \ldots, b_{8}\right\}
\end{aligned}
$$

To perform a single LFSR update (8 steps), we only need to calculate new values for one register, hi_new=update (lo, hi) while the other register update is a copy lo_new=hi.

Let $\mathrm{gA}=0 \times 090 \mathrm{f}$ represents the generating polynomial $g^{A}(\alpha)$ of the field $\mathbb{F}_{2^{16}}^{A^{-}}$, without the term $\alpha^{16}$. Then, multiplication of $x$ by $\alpha$ in $\mathbb{F}_{2^{16}}^{A}$ can be done as follows: we first shift $\mathrm{x}<<1$, then, based on the 15 th bit of the original $\mathrm{x}$, we XOR the result with gA. This may be done with only 4 instructions, using 16 -bit values

mul_alpha(uint16 $\mathrm{x}$, uint16 $\mathrm{gA}):=(\mathrm{x}<<1)$ xor $(($ signed int16) $\mathrm{x}>>15)$ and $\mathrm{gA})$

Note that the condition wether to xor with gA or not is implemented with the help of the 16 -bit mask $=$ (signed int16) $\mathrm{x} \gg>15$, where the mask is created by the arithmetical shift of the signed $\mathrm{x}$ to the right by 15 positions. The arithmetical shift to the right results in propagation of the sign (15th) bit, thus forming the mask either 0xffff in case the bit 15 was 1 , or $0 \times 0000$, otherwise.

The above trick can be applied to the combined 256 -bit vector $10=\left(b_{7}, \ldots, b_{0}, a_{7}, \ldots, a_{0}\right)$ to multiply the first half with $\alpha$ from the first base field $\mathbb{F}_{2^{16}}^{A}$ and the high part with $\beta$ from the second base field $\mathbb{F}_{2^{16}}^{B}$, simultaneously. Here we need to use _mm256_srai_epi16() that performs arithmetical shift to the right of 16 16-bit signed integers represented in the combined 256-bit register 1o. Obviously, the and operand should be done with the constant where the low $8 \times 16$-bit values are $\mathrm{gA}=0 \times 990 \mathrm{f}$ and the second half contains $\mathrm{gB}=0 \mathrm{xc} 963$.

A similar idea is applied for multiplication of hi by $\alpha^{-1}$ and $\beta^{-1}$. In our reference implementation we found the way with only 4 instructions with the help of a non-trivial intrinsic_mm256_sign_epi16() - however, if that intrinsic is not available then there is an alternative solution with 5 instructions.

The results of the above two steps should be XORed together with the values at tap offsets 1 and 3 for LFSRs A and B, respectively. The latter part is just byte shuffling that can be done with_mm256_blend_epi32() and_mm256_alignr_epi8(), three instructions in total.

\section{Software performance evaluation}

In this section we give software performance benchmarks of SNOW-V-(GCM), implemented by us in C++ (Visual Studio 2017) utilizing AVX2/AES-NI/PCLMULQDQ intrinsics.

\footnotetext{
${ }^{1}$ This intrinsic is intended for addition of signed integers but because most CPUs use two's complement representation for negative numbers, it will produce the correct results also for the unsigned addition needed in SNOW-V.
} 
All performance tests were carried out on a user-grade laptop with Intel i7-8650U CPU $@ 1.90 \mathrm{GHz}$ with Turbo Boost up to @ $4.20 \mathrm{GHz}$, testing each algorithm on a single process/thread and with various lengths of the input plaintext. Before each encryption process, we perform a key/IV setup procedure. In the first place, we should compare SNOW-V with AES since it demonstrates the fastest speed on commodity CPUs with available AES-NI instructions set. Perhaps, the second best choice algorithm, that is in various places serves as a backup for AES, is ChaCha20, and we should compare with that as well. We also include our best possible implementation of SNOW-3G in order to demonstrate the advantage of the new member of the SNOW family of stream ciphers.

For a fair and most challenging comparison we have downloaded the latest OpenSSL (3.0.0-dev, 2019-04-01) sources, and built it with the latest NASM and Visual Studio 2017 for that certain native x64 machine, with most possible optimizations switched on.

Implementations of the algorithms AES-256-(CBC, CTR, GCM) and ChaCha20(Poly1305) in OpenSSL are the most recent and highly optimized assembly codes that utilize AVX2/AES-NI/PCLMULQDQ, instructions stitching, and other best practice optimization techniques. OpenSSL's command line tool was used for performance evaluation of the selected algorithms; it runs an algorithm for a chosen number of seconds and delivers the number of bytes per second processed, out of which we derive the speed in Gigabits/sec (Gbps).

In order to fully align our own measurements of SNOW-V with the numbers given by OpenSSL, we actually extracted and adopted the benchmarking code from OpenSSL's sources and did exactly the same way of measurements of SNOW-V. To negotiate pitfalls from the system and the OS, we benchmarked every considered algorithm several times, for 1-3 seconds, then picked the best values (a similar method is used in SUPERCOP benchmarking approach). The results are presented in Table 3 .

Table 3: Performance comparison of SNOW-V-(GCM) and best OpenSSL's algorithms. Performance values are given in Gbps.

\begin{tabular}{|c|c|c|c|c|c|c|c|}
\hline Encryption only & \multicolumn{7}{|c|}{ Size of input plaintext (bytes) } \\
\cline { 2 - 8 } & 16384 & 8192 & 4096 & 2048 & 1024 & 256 & 64 \\
\hline SNOW-3G-128 (C++) & 9.22 & 9.07 & 8.89 & 8.50 & 7.81 & 5.38 & 2.37 \\
AES-256-CBC (asm) & 8.50 & 8.50 & 8.49 & 8.48 & 8.42 & 8.11 & 7.07 \\
ChaCha20 (asm) & 26.53 & 26.41 & 26.29 & 25.86 & 24.99 & 11.80 & 5.61 \\
AES-256-CTR (asm) & 35.06 & 34.82 & 34.16 & 32.94 & 30.95 & 22.67 & 11.32 \\
SNOW-V (C++) & $\mathbf{5 8 . 2 5}$ & 56.98 & 54.60 & 50.70 & 45.28 & 26.37 & 9.85 \\
\hline \hline AEAD mode & \multicolumn{7}{|c|}{} \\
\hline ChaCha20-Poly1305 (asm) & 18.46 & 18.24 & 18.16 & 17.54 & 16.99 & 8.98 & 4.29 \\
AES-256-GCM (asm) & 34.42 & 33.86 & 32.74 & 30.49 & 27.22 & 17.32 & 8.54 \\
SNOW-V-GCM (C++) & $\mathbf{3 8 . 9 1}$ & 37.66 & 34.86 & 30.71 & 26.16 & 13.93 & 5.16 \\
\hline
\end{tabular}

For a large plaintext, SNOW-V outperforms AES-256-CBC by around 6.5 times, even though AES-256-CBC is implemented in an optimized assembly code with AES-NI. SNOW-V is also 2 times faster than ChaCha20. An encryption in AES-256-CTR can be done in "parallel", so that the technique called "instructions interleaving" makes it possible to speed up a lot. Even here, SNOW-V is $66 \%$ faster than AES-256-CTR.

We would like to note that running an algorithm for even 1 second includes a lot of system overhead such as OS's scheduler, switching to other hundreds of OS's processes and services, switching CPUs' contexts and affinity for load balancing, etc. When we tried to measure SNOW-V for a very small fraction of a second (basically, measuring a single encryption), we have seen the speed goes up to 67Gbps (for encryption of 16384 bytes), which indicates that the OS's overhead is a significant factor.

One could notice from our measurements that AEAD mode of OpenSSL's AES-256- 
GCM is almost for "free" (35.06Gbps vs. 34.42Gbps). This was achieved by careful instructions interleaving and stitching techniques done in their optimized assembly code. In case of SNOW-V-GCM we, however, did not get GHASH for "free" in our $\mathrm{C}++$ implementation $(58.25 \mathrm{Gbps}$ vs. $38.91 \mathrm{Gbps})$, but we think that an optimized assembly implementation of SNOW-V-(GCM) could potentially give a better result.

AVX512 is a new set of intrinsics utilizing wider 512-bit registers, and a subset of the AVX512 instructions is currently only available on high-end Intel CPUs. In this new set of intrinsics, there is an instruction to perform 4 AES encryption rounds in parallel _mm512_aesenc_epi128(), which would speed up AES-256-CTR by around x4 times.

For SNOW-V it will mainly reduce the number of instructions, as several operations in both the LFSR and the FSM can be combined in a single new AVX512 instruction. A first approximation is that the number of instructions will be approximately halved, but we need to evaluate SNOW-V on a full AVX512 implementation first. Since SNOW-V would only use half of the 512-bit wide registers, the second half could be used to perform another SNOW-V instance in parallel, with its own key and IV. Thus, as a rough estimate, the speed of SNOW-V could be increased by 2-4 times.

We also did small tests for ARM NEON implementations of SNOW-V and AES on an Apple A11 ARM processor ${ }^{2}$. Note that ARM architectures for devices are very different from Intel desktop/server architectures in the sense that there is not a standardized implementation of the SoC. It is up to the SoC designer to decide on e.g. cache configurations. At least, this test gives some indication of relative performance. The SNOW-V implementation is a single threaded code using NEON intrinsics in C, and the AES-CTR implementation is a single threaded assembly code from OpenSSL 1.1.1c. The results are presented in Table 4.

Table 4: Performance comparison of SNOW-V and AES-CTR on an Apple A11 ARM processor. Performance values are given in Gbps.

\begin{tabular}{|c|c|c|c|c|c|c|c|}
\hline & \multicolumn{7}{|c|}{ Size of input plaintext (bytes) } \\
\cline { 2 - 8 } & 16384 & 8192 & 4096 & 2048 & 1024 & 256 & 64 \\
\hline SNOW-V (C) & 23.59 & 23.24 & 22.38 & 21.31 & 19.39 & 12.31 & 5.0 \\
AES-CTR (asm) & 15.97 & 15.87 & 15.59 & 15.08 & 14.34 & 10.62 & 5.04 \\
\hline
\end{tabular}

\section{Conclusions}

A new 128-bit stream cipher called SNOW-V is presented. It follows the design principles of the previous ciphers in the SNOW family, but leverages the AES round function instruction support found in many modern CPUs. In a single thread implementation in software, SNOW-V outperforms AES in all comparable modes of operation for plaintext lengths above 256 bytes. Basic cryptanalysis of the new design is presented and SNOW-V is argued to be resistant against these attacks. Finally, an AEAD mode of operation based on the well known GCM scheme is given. Test vectors and reference implementations are given in Appendix C, Appendix D, and Appendix E. We also provide a brief hardware evaluation of SNOW-V in Appendix F, including a 64-bit implementation utilizing a single AES core.

\section{Acknowledgments}

This work was in part financially supported by the Swedish Foundation for Strategic Research, grant RIT17-0005. Jing Yang is also supported by the scholarship from the National Digital Switching System Engineering and Technological Research Center, China.

\footnotetext{
2 Tests were run on an Apple iPhone X.
} 


\section{References}

[3GP] 3GPP. Work item on network functions virtualisation. http://www.3gpp. org/more/1584-nfv.

[Bab95] Steve Babbage. Improved "exhaustive search" attacks on stream ciphers. In European Convention on Security and Detection. IET, 1995.

[BG05] Olivier Billet and Henri Gilbert. Resistance of SNOW 2.0 against algebraic attacks. In Alfred Menezes, editor, Topics in Cryptology - CT-RSA 2005, volume 3376 of Lecture Notes in Computer Science, pages 19-28, San Francisco, CA, USA, February 14-18, 2005. Springer, Heidelberg, Germany.

[Bin] Bin Zhang et al. The ZUC-256 stream cipher. http://www.is.cas.cn/ ztzl2016/zouchongzhi/201801/W020180126529970733243.pdf.

[BS00] Alex Biryukov and Adi Shamir. Cryptanalytic time/memory/data tradeoffs for stream ciphers. In Tatsuaki Okamoto, editor, Advances in Cryptology ASIACRYPT 2000, volume 1976 of Lecture Notes in Computer Science, pages 1-13, Kyoto, Japan, December 3-7, 2000. Springer, Heidelberg, Germany.

[BSW01] Alex Biryukov, Adi Shamir, and David Wagner. Real time cryptanalysis of A5/1 on a PC. In Bruce Schneier, editor, Fast Software Encryption - FSE 2000, volume 1978 of Lecture Notes in Computer Science, pages 1-18, New York, NY, USA, April 10-12, 2001. Springer, Heidelberg, Germany.

[BW99] Alex Biryukov and David Wagner. Slide attacks. In Lars R. Knudsen, editor, Fast Software Encryption - FSE'99, volume 1636 of Lecture Notes in Computer Science, pages 245-259, Rome, Italy, March 24-26, 1999. Springer, Heidelberg, Germany.

[CHJ02] D. Coppersmith, S. Halevi, and C.S. Jutla. Cryptanalysis of stream ciphers with linear masking. In M. Yung, editor, Advances in Cryptology-CRYPTO 2002, volume 2442, pages 515-532, 2002.

[CJM02] Philippe Chose, Antoine Joux, and Michel Mitton. Fast correlation attacks: An algorithmic point of view. In Lars R. Knudsen, editor, Advances in Cryptology EUROCRYPT 2002, volume 2332 of Lecture Notes in Computer Science, pages 209-221, Amsterdam, The Netherlands, April 28 - May 2, 2002. Springer, Heidelberg, Germany.

[CJS01] Vladimor V. Chepyzhov, Thomas Johansson, and Ben J. M. Smeets. A simple algorithm for fast correlation attacks on stream ciphers. In Bruce Schneier, editor, Fast Software Encryption - FSE 2000, volume 1978 of Lecture Notes in Computer Science, pages 181-195, New York, NY, USA, April 10-12, 2001. Springer, Heidelberg, Germany.

[CKPS00] Nicolas Courtois, Alexander Klimov, Jacques Patarin, and Adi Shamir. Efficient algorithms for solving overdefined systems of multivariate polynomial equations. In Bart Preneel, editor, Advances in Cryptology - EUROCRYPT 2000, volume 1807 of Lecture Notes in Computer Science, pages 392-407, Bruges, Belgium, May 14-18, 2000. Springer, Heidelberg, Germany.

[DK08] Orr Dunkelman and Nathan Keller. Treatment of the initial value in timememory-data tradeoff attacks on stream ciphers. Information Processing Letters, 107(5):133-137, 2008. 
[DS09] Itai Dinur and Adi Shamir. Cube attacks on tweakable black box polynomials. In Antoine Joux, editor, Advances in Cryptology - EUROCRYPT 2009, volume 5479 of Lecture Notes in Computer Science, pages 278-299, Cologne, Germany, April 26-30, 2009. Springer, Heidelberg, Germany.

[Dwo07] Morris J. Dworkin. Sp 800-38d. recommendation for block cipher modes of operation: Galois/counter mode (gcm) and gmac. Technical report, Gaithersburg, MD, United States, 2007.

[EJ01] Patrik Ekdahl and Thomas Johansson. SNOW - a new stream cipher. In Proceedings of First Open NESSIE Workshop, KU-Leuven, 2001.

[EJ02] Patrik Ekdahl and Thomas Johansson. A New Version of the Stream Cipher SNOW. In Kaisa Nyberg and Howard M. Heys, editors, Selected Areas in Cryptography, volume 2595 of Lecture Notes in Computer Science, pages 47-61. Springer, 2002.

[EJT07] Håkan Englund, Thomas Johansson, and Meltem Sönmez Turan. A framework for chosen IV statistical analysis of stream ciphers. In K. Srinathan, C. Pandu Rangan, and Moti Yung, editors, Progress in Cryptology - INDOCRYPT 200\%: 8th International Conference in Cryptology in India, volume 4859 of Lecture Notes in Computer Science, pages 268-281, Chennai, India, December 9-13, 2007. Springer, Heidelberg, Germany.

[Gol97] Jovan Dj Golić. Cryptanalysis of alleged A5 stream cipher. In International Conference on the Theory and Applications of Cryptographic Techniques, pages 239-255. Springer, 1997.

[HJB09] Martin Hell, Thomas Johansson, and Lennart Brynielsson. An overview of distinguishing attacks on stream ciphers. Cryptography and Communications, 1(1):71-94, 2009.

[HK18] Matthias Hamann and Matthias Krause. On stream ciphers with provable beyond-the-birthday-bound security against time-memory-data tradeoff attacks. Cryptography and Communications, 10(5):959-1012, 2018.

[HR02] P. Hawkes and G.G. Rose. Guess-and-determine attacks on SNOW. In K. Nyberg and H. Heys, editors, Selected Areas in Cryptography-SAC 2002, volume 2595, pages 37-46, 2002.

[HS05] Jin Hong and Palash Sarkar. New applications of time memory data tradeoffs. In International Conference on the Theory and Application of Cryptology and Information Security, pages 353-372. Springer, 2005.

[Int18] Intel Corporation. Intel Intrinsics Guide. Technical report, 2018. https: //software.intel.com/sites/landingpage/IntrinsicsGuide/.

[ITU17] ITU. Minimum requirements related to technical performance for IMT-2020 radio interface(s). Version 1.0, ITU, 2017. https://www . itu.int/pub/R-REP-M. 2410-2017.

[KY11] Aleksandar Kircanski and Amr M Youssef. On the sliding property of SNOW 3G and SNOW 2.0. IET Information Security, 5(4):199-206, 2011.

[Lai94] Xuejia Lai. Higher order derivatives and differential cryptanalysis. In Communications and Cryptography, pages 227-233. Springer, 1994. 
[Max19] Alexander Maximov. AES MixColumn with 92 XOR gates. Cryptology ePrint Archive, Report 2019/833, 2019. https://eprint.iacr.org/2019/833.

[Mj06] O Saarinen Markku-juhani. Chosen-IV statistical attacks on eSTREAM stream ciphers. In eSTREAM, ECRYPT Stream Cipher Project, Report 2006/013. Citeseer, 2006.

[NW06] Kaisa Nyberg and Johan Wallén. Improved linear distinguishers for SNOW 2.0. In Matthew J. B. Robshaw, editor, Fast Software Encryption - FSE 2006, volume 4047 of Lecture Notes in Computer Science, pages 144-162, Graz, Austria, March 15-17, 2006. Springer, Heidelberg, Germany.

[oST01] National Institute of Standards and Technology. Advanced encryption standard. NIST FIPS PUB 197, 2001.

[RMTA18] Arash Reyhani-Masoleh, Mostafa Taha, and Doaa Ashmawy. Smashing the implementation records of aes s-box. IACR Transactions on Cryptographic Hardware and Embedded Systems, 2018(2):298-336, May 2018. https://tches . iacr.org/index.php/TCHES/article/download/884/835/.

[SA318] 3GPP SA3. TR 33.841 study on supporting 256-bit algorithms for 5G., 2018. https://portal.3gpp.org/desktopmodules/Specifications/ SpecificationDetails . aspx? specificationId $=3422$.

[SAG06] SAGE. Specification of the 3GPP Confidentiality and Integrity Algorithms UEA2 \& UIA2. Version 1.1, ETSI/SAGE, 2006. https://www.gsma.com/ aboutus/wp-content/uploads/2014/12/snow3gspec.pdf.

[SAG11] SAGE. Specification of the 3GPP Confidentiality and Integrity Algorithms 128-EEA3 \& 128-EIA3. document 2: ZUC specification. Version 1.6, ETSI/SAGE, 2011. https://www.gsma.com/aboutus/wp-content/uploads/ 2014/12/eea3eia3zucv16.pdf.

[Sam00] Samsung Electronics Co., Ltd. STD90/MDL90 $0.35 \mu \mathrm{m}$ 3.3V CMOS Standard Cell Library for Pure Logic/MDL Products Databook, 2000. https://www.digchip.com/datasheets/download_datasheet.php? $i d=935791$ \&part - number $=$ STD90.

[Sta10] Paul Stankovski. Greedy distinguishers and nonrandomness detectors. In Guang Gong and Kishan Chand Gupta, editors, Progress in Cryptology INDOCRYPT 2010: 11th International Conference in Cryptology in India, volume 6498 of Lecture Notes in Computer Science, pages 210-226, Hyderabad, India, December 12-15, 2010. Springer, Heidelberg, Germany.

[Sta13] Paul Stankovski. Cryptanalysis of Selected Stream Ciphers, volume 50. Department of Electrical and Information Technology, Lund University, 2013.

[SWW16] Ling Sun, Wei Wang, and Meiqin Wang. Milp-aided bit-based division property for primitives with non-bit-permutation linear layers. IACR Cryptology ePrint Archive, 2016:811, 2016.

[SWW17] Ling Sun, Wei Wang, and Meiqin Wang. Automatic search of bit-based division property for ARX ciphers and word-based division property. In Tsuyoshi Takagi and Thomas Peyrin, editors, Advances in Cryptology - ASIACRYPT 2017, Part I, volume 10624 of Lecture Notes in Computer Science, pages 128-157, Hong Kong, China, December 3-7, 2017. Springer, Heidelberg, Germany. 
[TIHM17] Yosuke Todo, Takanori Isobe, Yonglin Hao, and Willi Meier. Cube attacks on non-blackbox polynomials based on division property. In Jonathan Katz and Hovav Shacham, editors, Advances in Cryptology - CRYPTO 2017, Part III, volume 10403 of Lecture Notes in Computer Science, pages 250-279, Santa Barbara, CA, USA, August 20-24, 2017. Springer, Heidelberg, Germany.

[Tod15] Yosuke Todo. Structural evaluation by generalized integral property. In Elisabeth Oswald and Marc Fischlin, editors, Advances in Cryptology - EUROCRYPT 2015, Part I, volume 9056 of Lecture Notes in Computer Science, pages 287-314, Sofia, Bulgaria, April 26-30, 2015. Springer, Heidelberg, Germany.

[UMHA16] Rei Ueno, Sumio Morioka, Naofumi Homma, and Takafumi Aoki. A high throughput/gate aes hardware architecture by compressing encryption and decryption datapaths - toward efficient cbc-mode implementation. Cryptology ePrint Archive, Report 2016/595, 2016. https://eprint.iacr.org/2016/ 595.

[Vie07] Michael Vielhaber. Breaking ONE.FIVIUM by AIDA an algebraic IV differential attack. Cryptology ePrint Archive, Report 2007/413, 2007. http: //eprint.iacr.org/2007/413.

$\left[\mathrm{WHT}^{+}\right.$18] Qingju Wang, Yonglin Hao, Yosuke Todo, Chaoyun Li, Takanori Isobe, and Willi Meier. Improved division property based cube attacks exploiting algebraic properties of superpoly. In Annual International Cryptology Conference, pages 275-305. Springer, 2018.

[ZXM15] Bin Zhang, Chao Xu, and Willi Meier. Fast correlation attacks over extension fields, large-unit linear approximation and cryptanalysis of SNOW 2.0. In Rosario Gennaro and Matthew J. B. Robshaw, editors, Advances in Cryptology - CRYPTO 2015, Part I, volume 9215 of Lecture Notes in Computer Science, pages 643-662, Santa Barbara, CA, USA, August 16-20, 2015. Springer, Heidelberg, Germany. 


\section{A Remarks about the maximum period of the LFSR struc- ture}

We can denote the LFSR state at time $t \geq 0$ as

$$
S^{(t)}=\left(a_{0}^{(t)}, a_{1}^{(t)}, \ldots, a_{14}^{(t)}, a_{15}^{(t)}, b_{0}^{(t)}, b_{1}^{(t)}, \ldots, b_{14}^{(t)}, b_{15}^{(t)}\right)
$$

with 32 16-bit cells, i.e., 512 bits in total. If we consider the binary representation of the state, then the next state at $t+1, S^{(t+1)}$ can be written as,

$$
S^{(t+1)}=S^{(t)} M
$$

where $M$ is the $512 \times 512$ state transition matrix.

Every cell of the next state except $a_{15}^{(t+1)}, b_{15}^{(t+1)}$ is determined by a shift from the neighboring cell, that is $a_{i}^{(t+1)}=a_{i+1}^{(t)}, b_{i}^{(t+1)}=b_{i+1}^{(t)}$ for $i=0,1, \ldots 14$, and the corresponding binary state transition submatrices for such update are identity matrix $M_{I}$ with size $16 \times 16$. As for $a_{15}, b_{15}$, we can rewrite them in the polynomial form. Suppose the bases for finite fields $\mathbb{F}_{2^{16}}^{A}$ and $\mathbb{F}_{2^{16}}^{B}$ are respectively $\left(1, \alpha, \ldots, \alpha^{15}\right),\left(1, \beta, \ldots, \beta^{15}\right)$, then every state element can be expressed as a polynomial corresponding to the two bases.

For instance, a certain element $e \in \mathbb{F}_{2^{16}}^{A}$ can be interpreted as $e=e_{0}+e_{1} \alpha+, \ldots,+e_{14} \alpha^{14}+$ $e_{15} \alpha^{15}$, where $e_{i}$ denotes the value at the $i$-th position of $e$. Then,

$$
e \alpha \bmod g^{A}(\alpha)=\left(e_{15} \alpha^{16}+e_{14} \alpha^{15}+, \ldots,+e_{1} \alpha^{2}+e_{0} \alpha\right) \bmod g^{A}(\alpha)
$$

Since

$$
\alpha^{16} \bmod g^{A}(\alpha)=\alpha^{15}+\alpha^{12}+\alpha^{11}+\alpha^{8}+\alpha^{3}+\alpha^{2}+\alpha+1,
$$

e $\alpha \bmod g^{A}(\alpha)$ can be expanded and rearranged as,

$$
\begin{aligned}
& =e_{15}\left(\alpha^{15}+\alpha^{12}+\alpha^{11}+\alpha^{8}+\alpha^{3}+\alpha^{2}+\alpha+1\right)+e_{14} \alpha^{15}+, \ldots,+e_{1} \alpha^{2}+e_{0} \alpha \\
& =\left(e_{15}+e_{14}\right) \alpha^{15}+e_{13} \alpha^{14}+e_{12} \alpha^{13}+\left(e_{15}+e_{11}\right) \alpha^{12}+\left(e_{15}+e_{10}\right) \alpha^{11}+ \\
& e_{9} \alpha^{10}+e_{8} \alpha^{9}+\left(e_{15}+e_{7}\right) \alpha^{8}+e_{6} \alpha^{7}+e_{5} \alpha^{6}+e_{4} \alpha^{5}+e_{3} \alpha^{4}+\left(e_{15}+e_{2}\right) \alpha^{3} \\
& +\left(e_{15}+e_{1}\right) \alpha^{2}+\left(e_{15}+e_{0}\right) \alpha+e_{15} \\
& =\left(e_{0}, e_{1}, \ldots, e_{15}\right) M_{\alpha}\left(1, \alpha, \ldots, \alpha^{15}\right)^{\mathrm{T}}
\end{aligned}
$$

From which we can deduce the matrix

$$
M_{\alpha}=\left(\begin{array}{cccccccccccccccc}
0 & 1 & 0 & 0 & 0 & 0 & 0 & 0 & 0 & 0 & 0 & 0 & 0 & 0 & 0 & 0 \\
0 & 0 & 1 & 0 & 0 & 0 & 0 & 0 & 0 & 0 & 0 & 0 & 0 & 0 & 0 & 0 \\
0 & 0 & 0 & 1 & 0 & 0 & 0 & 0 & 0 & 0 & 0 & 0 & 0 & 0 & 0 & 0 \\
0 & 0 & 0 & 0 & 1 & 0 & 0 & 0 & 0 & 0 & 0 & 0 & 0 & 0 & 0 & 0 \\
0 & 0 & 0 & 0 & 0 & 1 & 0 & 0 & 0 & 0 & 0 & 0 & 0 & 0 & 0 & 0 \\
0 & 0 & 0 & 0 & 0 & 0 & 1 & 0 & 0 & 0 & 0 & 0 & 0 & 0 & 0 & 0 \\
0 & 0 & 0 & 0 & 0 & 0 & 0 & 1 & 0 & 0 & 0 & 0 & 0 & 0 & 0 & 0 \\
0 & 0 & 0 & 0 & 0 & 0 & 0 & 0 & 1 & 0 & 0 & 0 & 0 & 0 & 0 & 0 \\
0 & 0 & 0 & 0 & 0 & 0 & 0 & 0 & 0 & 1 & 0 & 0 & 0 & 0 & 0 & 0 \\
0 & 0 & 0 & 0 & 0 & 0 & 0 & 0 & 0 & 0 & 1 & 0 & 0 & 0 & 0 & 0 \\
0 & 0 & 0 & 0 & 0 & 0 & 0 & 0 & 0 & 0 & 0 & 1 & 0 & 0 & 0 & 0 \\
0 & 0 & 0 & 0 & 0 & 0 & 0 & 0 & 0 & 0 & 0 & 0 & 1 & 0 & 0 & 0 \\
0 & 0 & 0 & 0 & 0 & 0 & 0 & 0 & 0 & 0 & 0 & 0 & 0 & 1 & 0 & 0 \\
0 & 0 & 0 & 0 & 0 & 0 & 0 & 0 & 0 & 0 & 0 & 0 & 0 & 0 & 1 & 0 \\
0 & 0 & 0 & 0 & 0 & 0 & 0 & 0 & 0 & 0 & 0 & 0 & 0 & 0 & 0 & 1 \\
1 & 1 & 1 & 1 & 0 & 0 & 0 & 0 & 1 & 0 & 0 & 1 & 1 & 0 & 0 & 1
\end{array}\right)
$$


With the same method, we can also derive $M_{\alpha^{-1}}, M_{\beta}, M_{\beta^{-1}}$. Then we can rewrite the update for $a_{15}^{(t+1)}, b_{15}^{(t+1)}$ in a matrix form,

$$
\begin{aligned}
& a_{15}^{(t+1)}=b_{0}^{(t)} M_{I}+a_{0}^{(t)} M_{\alpha}+a_{1}^{(t)} M_{I}+a_{8}^{(t)} M_{\alpha^{-1}} \\
& b_{15}^{(t+1)}=a_{0}^{(t)} M_{I}+b_{0}^{(t)} M_{\beta}+b_{3}^{(t)} M_{I}+b_{8}^{(t)} M_{\beta^{-1}}
\end{aligned}
$$

Then the complete binary transition matrix for the LFSR update can be written as,

$$
\begin{aligned}
& \begin{array}{lllllllllllllll}
0 & 1 & \ldots & 7 & \ldots & 14 & 15 & \ldots & 18 & \ldots & 23 & \ldots & 30 & 31
\end{array} \\
& M_{\alpha} \quad M_{I} \\
& 1 M_{I} \\
& M_{I} \\
& M_{I} \quad M_{\alpha^{-1}} \\
& \begin{array}{lll}
\cdots & \cdots & \cdots \\
15 & & M_{I}
\end{array} \\
& 16 \\
& M_{I} \\
& \begin{array}{cccc} 
& & & M_{\beta} \\
& & & \\
& & & \\
& & & \\
& & M_{I} & M_{\beta^{-1}}
\end{array} \\
& 24 \\
& 31 \\
& M_{I}
\end{aligned}
$$

where every element in the $32 \times 32$ matrix is a $16 \times 16$ matrix and all the other empty places are $16 \times 16$ zero matrices. Then we can get the $512 \times 512$ transition matrix and some mathematical tools, such as Sagemath, can be employed to verify whether it is primitive. We employ the built-in function charpoly() in Sagemath to get the characteristic polynomial, which is,

$$
\begin{aligned}
& m(x)= \\
& x^{512}+x^{491}+x^{489}+x^{480}+x^{478}+x^{475}+x^{474}+x^{473}+x^{472}+x^{468}+x^{467}+ \\
& x^{466}+x^{464}+x^{455}+x^{453}+x^{452}+x^{445}+x^{444}+x^{443}+x^{441}+x^{438}+x^{437}+ \\
& x^{434}+x^{433}+x^{429}+x^{426}+x^{425}+x^{424}+x^{423}+x^{422}+x^{420}+x^{419}+x^{418}+ \\
& x^{417}+x^{416}+x^{415}+x^{410}+x^{409}+x^{407}+x^{405}+x^{404}+x^{402}+x^{394}+x^{393}+ \\
& x^{391}+x^{390}+x^{385}+x^{384}+x^{383}+x^{382}+x^{381}+x^{380}+x^{374}+x^{371}+x^{369}+ \\
& x^{368}+x^{367}+x^{366}+x^{365}+x^{363}+x^{361}+x^{360}+x^{358}+x^{357}+x^{354}+x^{351}+ \\
& x^{345}+x^{344}+x^{341}+x^{339}+x^{337}+x^{336}+x^{334}+x^{330}+x^{325}+x^{324}+x^{321}+ \\
& x^{317}+x^{315}+x^{314}+x^{313}+x^{311}+x^{310}+x^{309}+x^{308}+x^{307}+x^{305}+x^{302}+ \\
& x^{299}+x^{296}+x^{292}+x^{291}+x^{284}+x^{283}+x^{281}+x^{280}+x^{279}+x^{276}+x^{275}+ \\
& x^{273}+x^{271}+x^{267}+x^{264}+x^{263}+x^{262}+x^{260}+x^{259}+x^{258}+x^{257}+x^{256}+ \\
& x^{254}+x^{253}+x^{251}+x^{249}+x^{248}+x^{247}+x^{246}+x^{245}+x^{243}+x^{242}+x^{240}+ \\
& x^{238}+x^{236}+x^{229}+x^{225}+x^{218}+x^{217}+x^{216}+x^{215}+x^{214}+x^{209}+x^{208}+ \\
& x^{207}+x^{205}+x^{204}+x^{203}+x^{201}+x^{198}+x^{193}+x^{192}+x^{190}+x^{189}+x^{187}+ \\
& x^{186}+x^{185}+x^{180}+x^{178}+x^{176}+x^{173}+x^{170}+x^{169}+x^{167}+x^{165}+x^{164}+ \\
& x^{163}+x^{162}+x^{160}+x^{159}+x^{155}+x^{152}+x^{151}+x^{150}+x^{149}+x^{148}+x^{147}+ \\
& x^{145}+x^{144}+x^{142}+x^{141}+x^{136}+x^{134}+x^{131}+x^{126}+x^{125}+x^{123}+x^{122}+ \\
& x^{121}+x^{118}+x^{117}+x^{114}+x^{113}+x^{109}+x^{106}+x^{105}+x^{104}+x^{103}+x^{101}+ \\
& x^{100}+x^{96}+x^{95}+x^{94}+x^{91}+x^{87}+x^{86}+x^{85}+x^{83}+x^{82}+x^{81}+x^{78}+ \\
& x^{76}+x^{74}+x^{73}+x^{69}+x^{68}+x^{67}+x^{66}+x^{64}+x^{63}+x^{62}+x^{61}+x^{59}+ \\
& x^{56}+x^{54}+x^{53}+x^{50}+x^{49}+x^{47}+x^{42}+x^{38}+x^{36}+x^{35}+x^{33}+x^{25}+ \\
& x^{24}+x^{23}+x^{20}+x^{16}+x^{15}+x^{14}+x^{13}+x^{11}+x^{9}+x^{6}+x+1
\end{aligned}
$$

Then we can verify it primitive by Sagemath, which indicates the LFSR structure has the maximum period $2^{512}-1$. 
Then we get the following linear approximation of the FSM:

$$
\begin{aligned}
& \sum_{i=0}^{3}\left[L^{-1} z^{(t+1)} \oplus z^{(t)} \oplus z^{(t-1)}\right]_{i}=\sum_{i=0}^{3}(\underbrace{\left.S\left(\hat{R} 1_{i}\right) \oplus\left(\hat{R} 1_{i} \boxplus_{8} T 1_{i}^{(t)}\right) \oplus T 1_{i}^{(t)} \oplus T 1_{i}^{(t)}\right)}_{\text {Noise } N 1_{i}} \\
& \oplus \sum_{i=0}^{3}[\underbrace{\hat{R} 2_{i} \oplus \sum_{j=0}^{3} c_{4 i+j}\left[\left(\hat{R} 2_{4 i+j} \boxplus_{8}\left(\hat{R} 3_{4 i+j} \oplus \hat{X}_{i, j}\right) \boxplus_{8} \hat{Y}_{i, j}\right) \oplus \hat{X}_{i, j} \oplus \hat{Y}_{i, j} \oplus \hat{X}_{i, j} \oplus \hat{Y}_{i, j}\right]}_{\text {Linear part } C_{i} \text { of } N 2_{i}}] \\
& \oplus \sum_{i=0}^{3}\left[(\underbrace{\left.S^{-1}\left(\sum_{j=0}^{\sum_{j=0}^{3} c_{4 i+j} \hat{R} 2_{4 i+j}}\right) \boxplus_{8} T 1_{i}^{(t-1)}\right) \oplus S^{-1}(\underbrace{\sum_{j=0}^{3} c_{4 i+j} \hat{R} 3_{4 i+j}}_{\text {Linear part } B_{i} \text { of } N 2_{i}})}_{\text {Linear part } A_{i} \text { of } N 2_{i}}]\right.
\end{aligned}
$$

where $\hat{X}_{i, j}=T 2_{4 i+j}^{(t)}$ and $\hat{Y}_{i, j}=\left(\sigma^{-1} T 1^{(t+1)}\right)_{4 i+j}$.

In the above it now becomes clear that we need to compute byte-oriented distributions of two independent noise variables $N 1$ and $N 2$. The first noise is trivial to compute, while the second $N 2$ is a bit more complicated, but it can be computed with the technique mentioned in Section 3.4.1. I.e., for every $i$ we first compute 4 partial joint distributions $D_{i, j}\left(A_{i, j}\left|B_{i, j}\right| C_{i, j}\right)$ of linear parts $A|B| C$ for each $j=0 \ldots 3$, then we use Fast WalshHadamard Transform to perform the XOR-convolutions in order to get the complete 24-bit joint distribution $D_{i}\left(A_{i}\left|B_{i}\right| C_{i}\right)$ for all possible 32-bit input arguments running over $j=0,1,2,3$, for that certain $i$. Having that joint distribution $D_{i}\left(A_{i}\left|B_{i}\right| C_{i}\right)$ being computed it is then trivial to compute the 8-bit sub-noise distribution $N 2_{i}$ : we simply loop over all possible choices of $A_{i}, B_{i}, C_{i}, T 1_{i}^{(t-1)}$, and approximate $\ldots \boxplus_{8} T 1_{i}^{(t-1)}$ by adding that term outside of $S^{-1}$, near by the linear part A. I.e., we compute

$$
\operatorname{Pr}\left\{N 2_{i}=\left(S^{-1}\left(A_{i}\right) \boxplus_{8} T 1_{i}^{(t-1)}\right) \oplus T 1_{i}^{(t-1)} \oplus S^{-1}\left(B_{i}\right) \oplus C_{i}\right\}+=\frac{1}{2^{8}} \cdot D_{i}\left(A_{i}\left|B_{i}\right| C_{i}\right) .
$$

We repeat the above for each $i$, and the total noise $N 2$ is the XOR-convolution of the four independent sub-distributions $N 2_{i}$.

We would like to note that the first sub-distribution $N 2_{0}$ has a smaller bias since 4 bytes of the term $\hat{R} 2_{i}$ in the linear part $C$ of $N 2$ are added to $N 2_{0}$, while the remaining 3 sub-distributions are free from these terms. This means that $N 2_{0}$ includes 4 approximations of type $n 3$ with a smaller bias $\left(\approx 2^{-3.3}\right)$ than those of the types $n 1\left(\approx 2^{3.1}\right)$ and $n 2\left(\approx 2^{-2.9}\right)$. Our computation results are as follows: $\epsilon\left(N 2_{i=0}\right) \approx 2^{-53.828334}, \epsilon\left(N 2_{i=1 . .3}\right) \approx 2^{-30.382642}$, $\epsilon\left(N 1_{i=0 . .3}\right) \approx 2^{-2.920807}$, and

$$
\begin{aligned}
\epsilon\left(\sum_{i=0}^{3} N 1_{i}\right) & \approx 2^{-26.446376}, \\
\epsilon\left(\sum_{i=0}^{3} N 2_{i}\right) & \approx 2^{-187.562693}, \\
\epsilon\left(N_{t o t}\right) & \approx 2^{-214.848865}, \\
\epsilon\left(2 \times N_{t o t}\right) & \approx 2^{-429.674887} .
\end{aligned}
$$




\section{Test Vectors}

This section presents test vectors for SNOW-V with three different keys and IVs. The vectors are written with the least significant byte of the 128-bit word appearing to the left in the row. For the keys, the lower 128-bit part is written on the first row, followed by the high part on the second row.

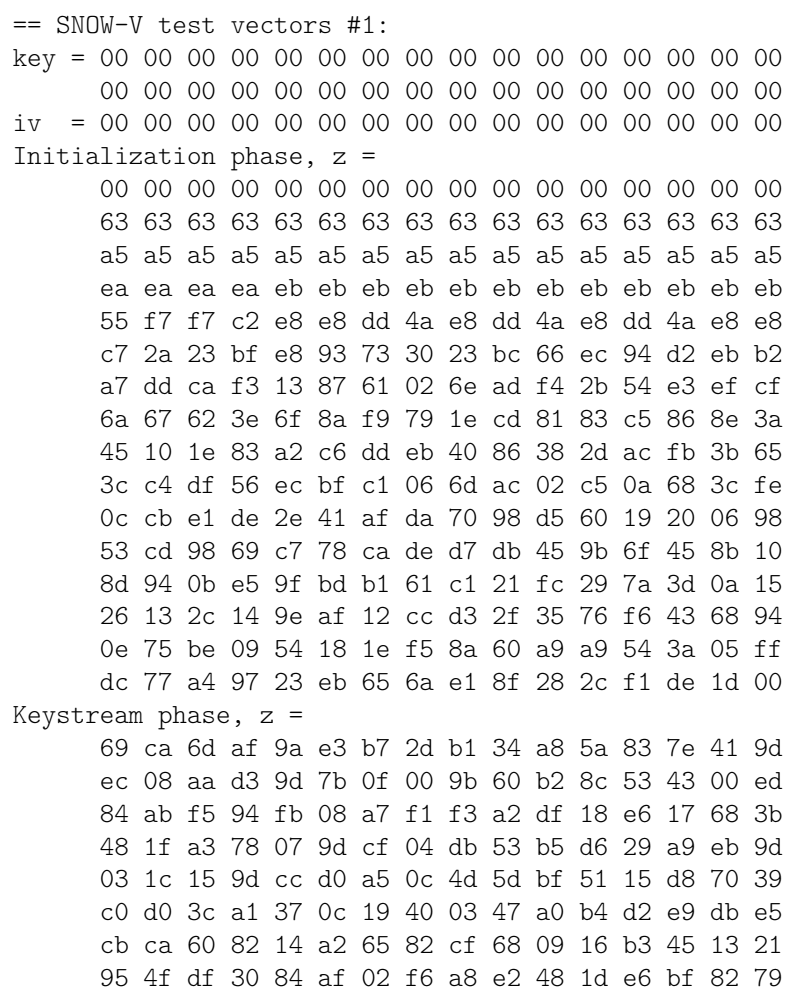

Listing 2: Test vectors for SNOW-V.

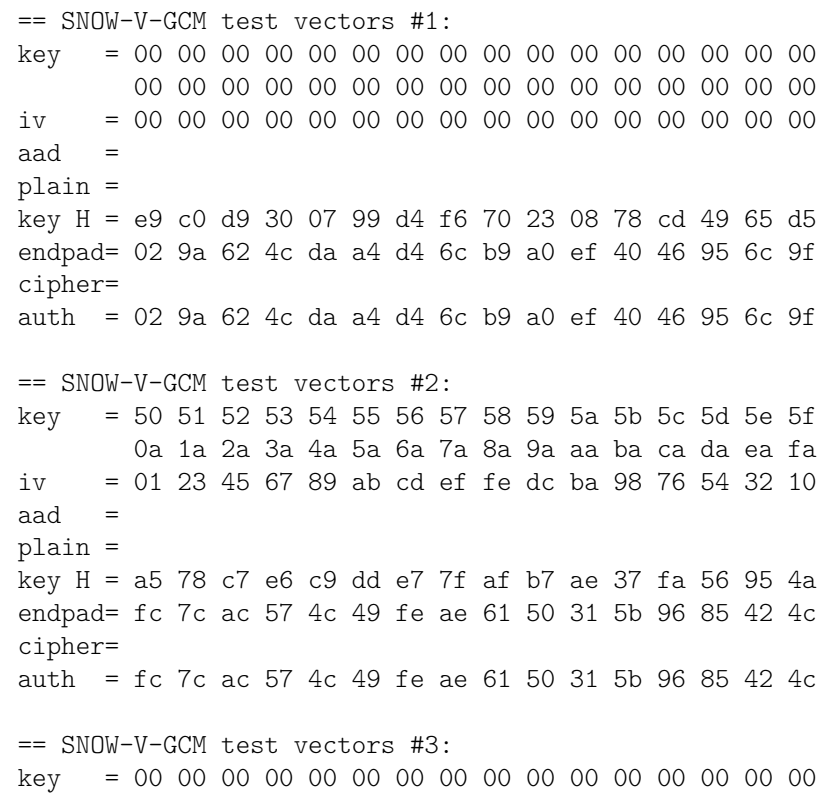




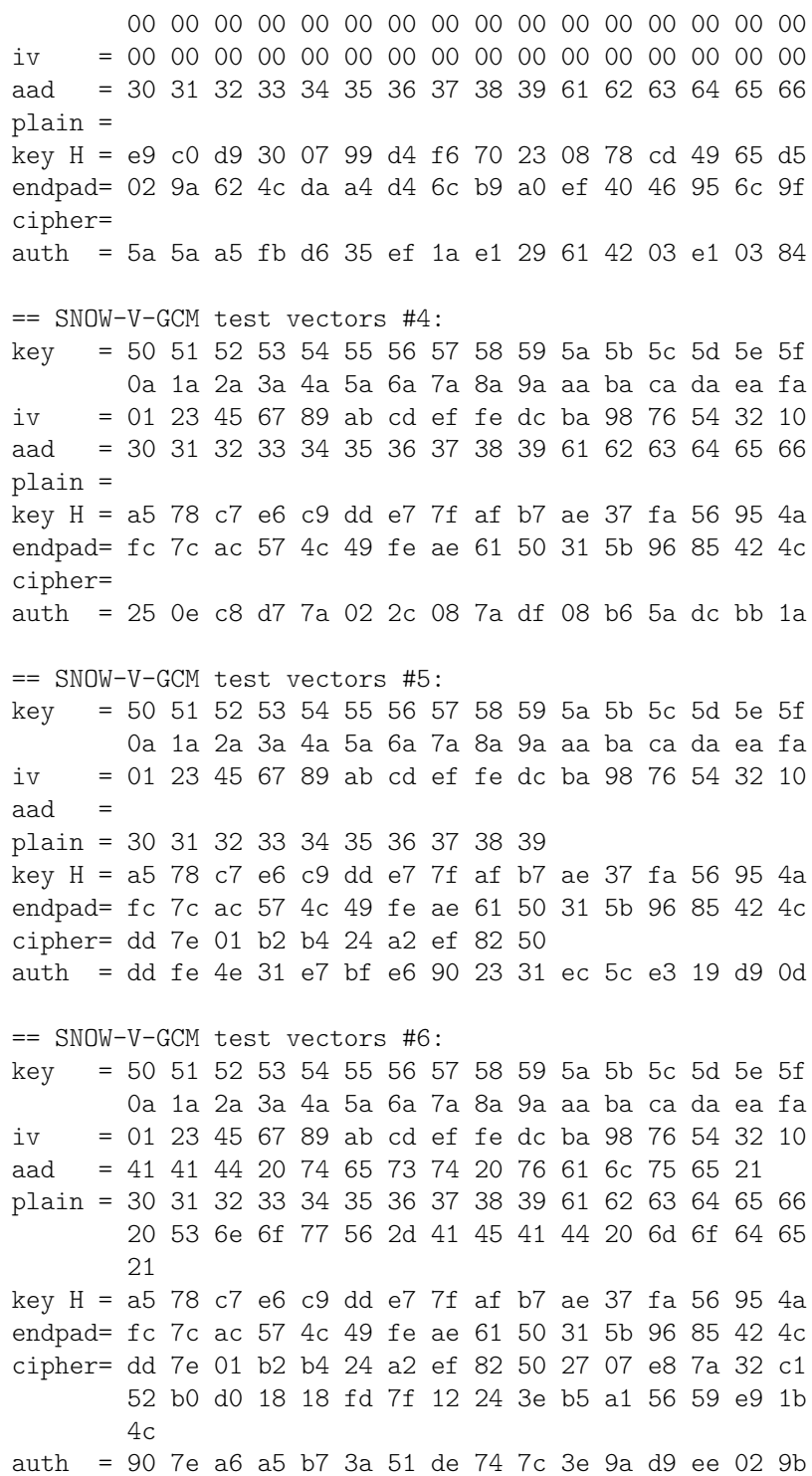

$\begin{array}{llllllllllllllll}00 & 00 & 00 & 00 & 00 & 00 & 00 & 00 & 00 & 00 & 00 & 00 & 00 & 00 & 00 & 00\end{array}$ iv $=\begin{array}{llllllllllllllll}00 & 00 & 00 & 00 & 00 & 00 & 00 & 00 & 00 & 00 & 00 & 00 & 00 & 00 & 00 & 00\end{array}$ aad $=\begin{array}{llllllllllllllll}30 & 31 & 32 & 33 & 34 & 35 & 36 & 37 & 38 & 39 & 61 & 62 & 63 & 64 & 65 & 66\end{array}$ plain $=$

key $\mathrm{H}=$ e9 $\mathrm{c0}$ d9 $30 \quad 07 \quad 99$ d4 f6 $70 \quad 23 \quad 08 \quad 78$ cd $49 \quad 65$ d5 endpad= $029 \mathrm{a} 624 \mathrm{c}$ da $\mathrm{a} 4 \mathrm{~d} 46 \mathrm{c}$ b9 a0 ef $4046956 \mathrm{c} 9 \mathrm{f}$ cipher $=$

auth $=5 \mathrm{a} 5 \mathrm{a}$ a5 fb d6 35 ef $1 \mathrm{a}$ e1 $2961 \quad 4203$ e1 0384 $==$ SNOW-V-GCM test vectors \#4:

key $=50515253545556575859$ 5a 5b 5c 5d 5e $5 f$ 0 a 1a 2 a $3 a$ 4a 5a $6 a$ 7a 8 a 9 a a ba ca da ea fa iv $=0123456789 \mathrm{ab} c d$ ef fe dc ba 9876543210 aad $=\begin{array}{llllllllllllllll}30 & 31 & 32 & 33 & 34 & 35 & 36 & 37 & 38 & 39 & 61 & 62 & 63 & 64 & 65 & 66\end{array}$ plain $=$

key $\mathrm{H}=$ a5 78 c7 e6 c9 dd e7 $7 f$ af b7 ae 37 fa 5695 4a endpad $=f c 7 c$ ac 57 4c 49 fe ae $6150315 b \quad 96 \quad 8542 \quad 4 c$ cipher $=$

auth $=250$ e c8 d7 $7 \mathrm{a} 02 \quad 2 \mathrm{c} 08 \quad 7 \mathrm{a}$ df 08 b6 $5 \mathrm{a}$ dc bb $1 \mathrm{a}$

$==$ SNOW-V-GCM test vectors \#5:

key $=50515253545556575859$ 5a 5b 5c 5d 5e 5f 0 a 1a 2 a $3 a$ 4a 5 a $6 a$ 7a 8 a $9 a$ aa ba ca da ea fa iv $=0123456789 \mathrm{ab}$ cd ef fe dc ba 9876543210 aad $=$

plain = $\begin{array}{llllllllll}30 & 31 & 32 & 33 & 34 & 35 & 36 & 37 & 38 & 39\end{array}$

key $\mathrm{H}=\mathrm{a} 5 \quad 78$ c7 e6 c9 dd e7 $7 \mathrm{f}$ af b7 ae $37 \mathrm{fa} 56954 \mathrm{a}$ endpad $=$ fc $7 \mathrm{c}$ ac 57 4c 49 fe ae 615031 5b $96 \quad 85424 c$ cipher $=$ dd 7 e 01 b2 b4 24 a2 ef 8250

auth = dd fe 4 e 31 e7 bf e6 $9023 \quad 31$ ec $5 c$ e3 19 d9 od

$==$ SNOW-V-GCM test vectors \#6:

key $=50515253545556575859$ 5a 5b 5c 5d 5e $5 f$ $0 a$ 1a 2a 3a 4a 5a 6a 7a 8a 9a aa ba ca da ea fa iv $=0123456789 \mathrm{ab} c d$ ef fe dc ba 9876543210 aad $=41414420 \quad 74 \quad 6573 \quad 742076 \quad 61 \quad 6 c \quad 75 \quad 65 \quad 21$ plain $=\begin{array}{llllllllllllllll}30 & 31 & 32 & 33 & 34 & 35 & 36 & 37 & 38 & 39 & 61 & 62 & 63 & 64 & 65 & 66\end{array}$ 20536 e $6 f 77 \quad 56$ 2d 4145414420 6d $6 f \quad 6465$ 21

key $\mathrm{H}=\mathrm{a} 5 \quad 78$ c7 e6 c9 dd e7 $7 \mathrm{f}$ af b7 ae 37 fa $5695 \quad 4 \mathrm{a}$ endpad= fc $7 c$ ac 57 4c 49 fe ae $\begin{array}{llllllll}61 & 50 & 31 & 5 b & 96 & 85 & 42 & 4 c\end{array}$ cipher $=$ dd 7 e 01 b2 b4 24 a2 ef $82 \quad 50 \quad 27 \quad 07$ e8 7 a 32 c1 52 b0 d0 1818 fd $7 f \quad 1224$ 3e b5 a1 5659 e9 1b $4 \mathrm{c}$

auth $=907 \mathrm{e}$ a6 a5 b7 3a 51 de 74 c 3 e 9a d9 ee 02 9b

Listing 3: Test vectors for SNOW-V-GCM. 


\section{SNOW-V 32-bit Reference Implementation in $\mathrm{C} / \mathrm{C}++$}

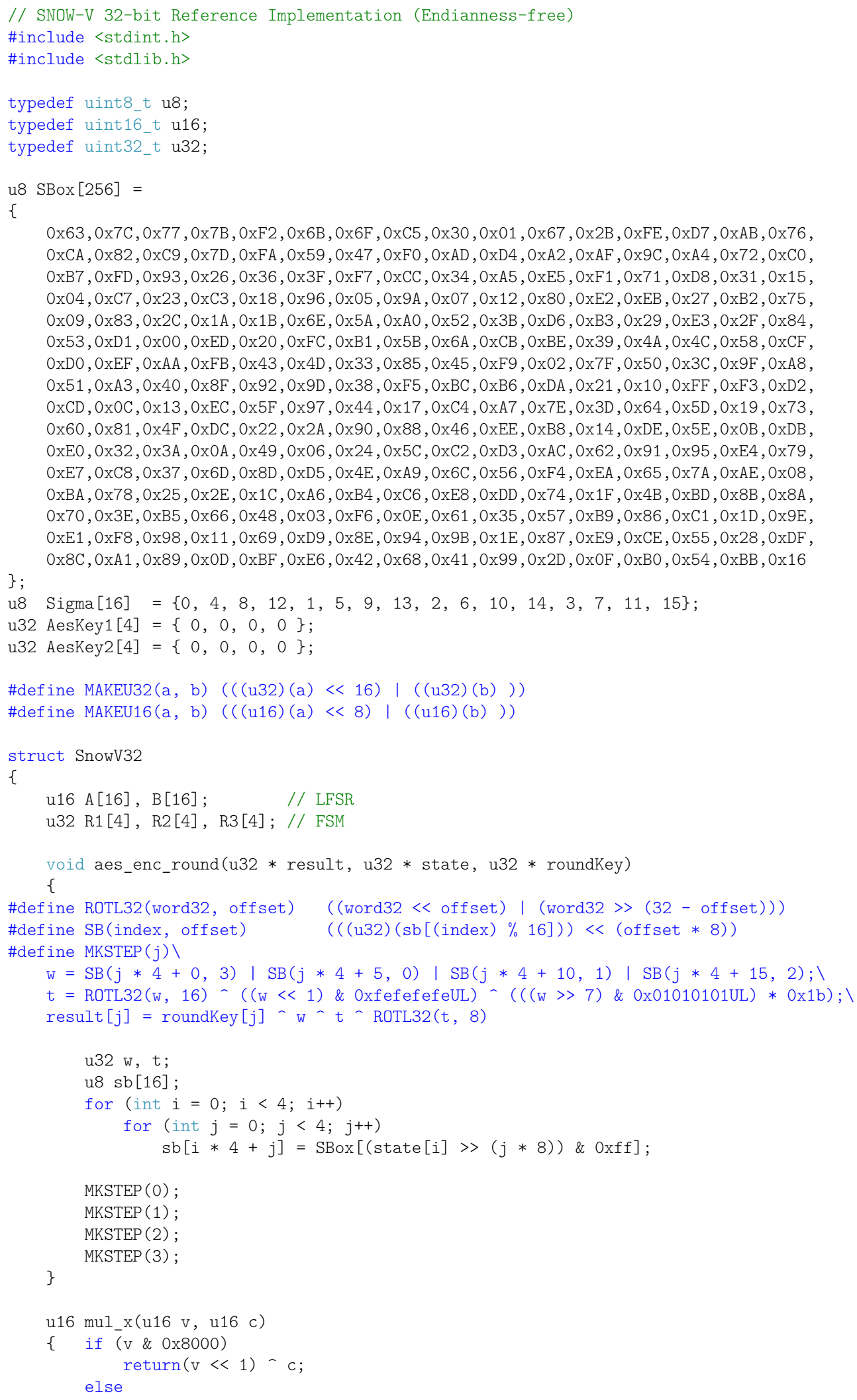




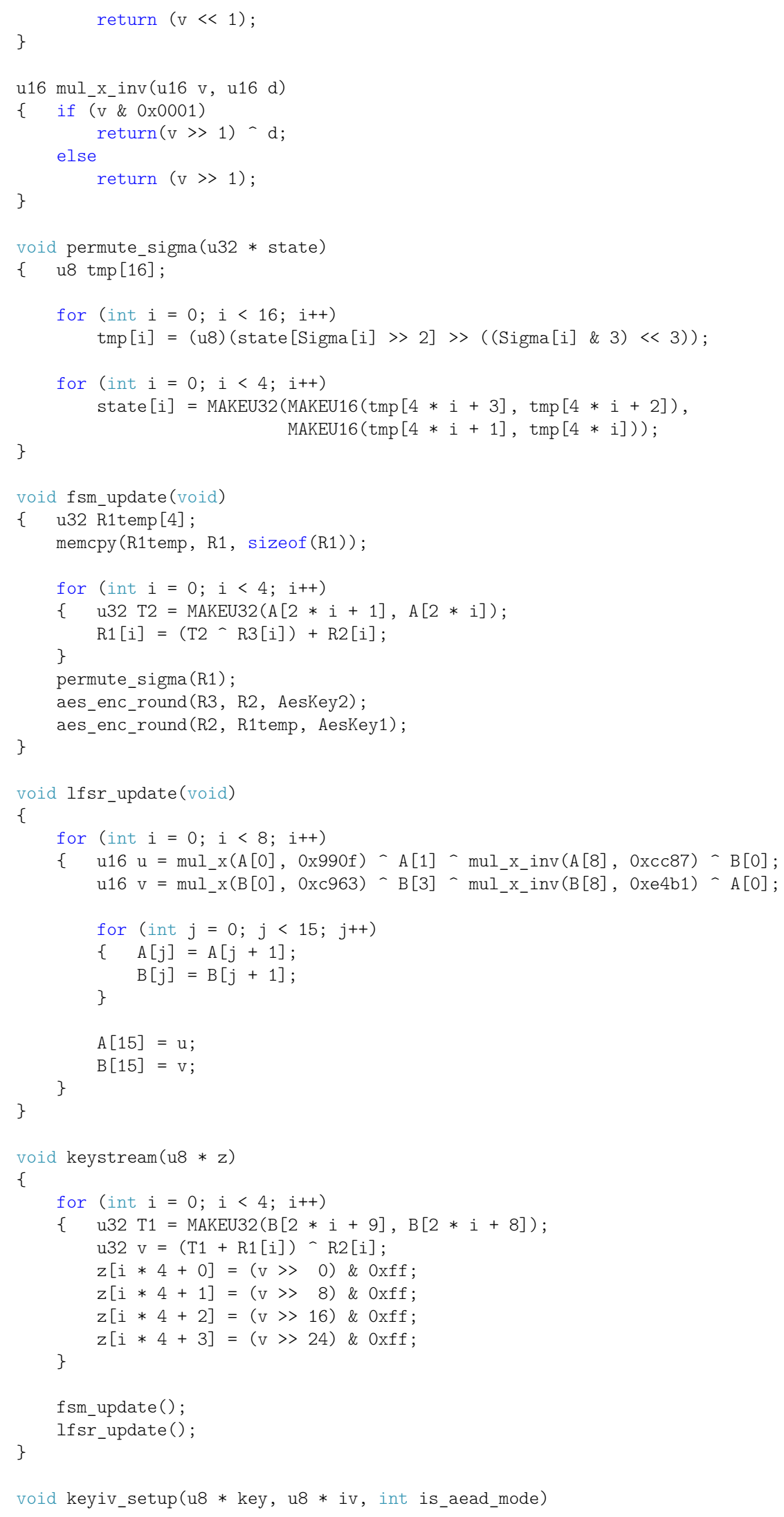




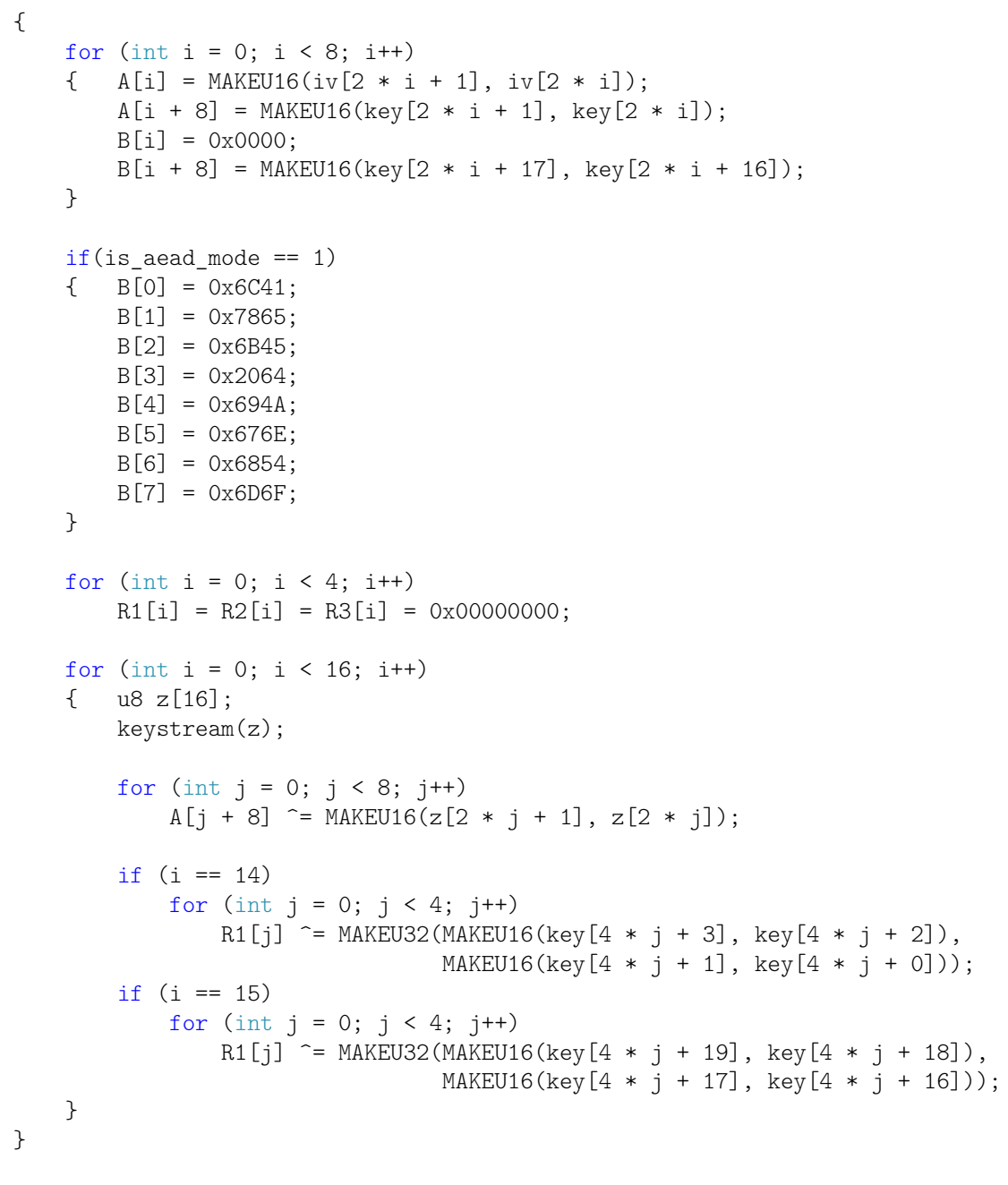




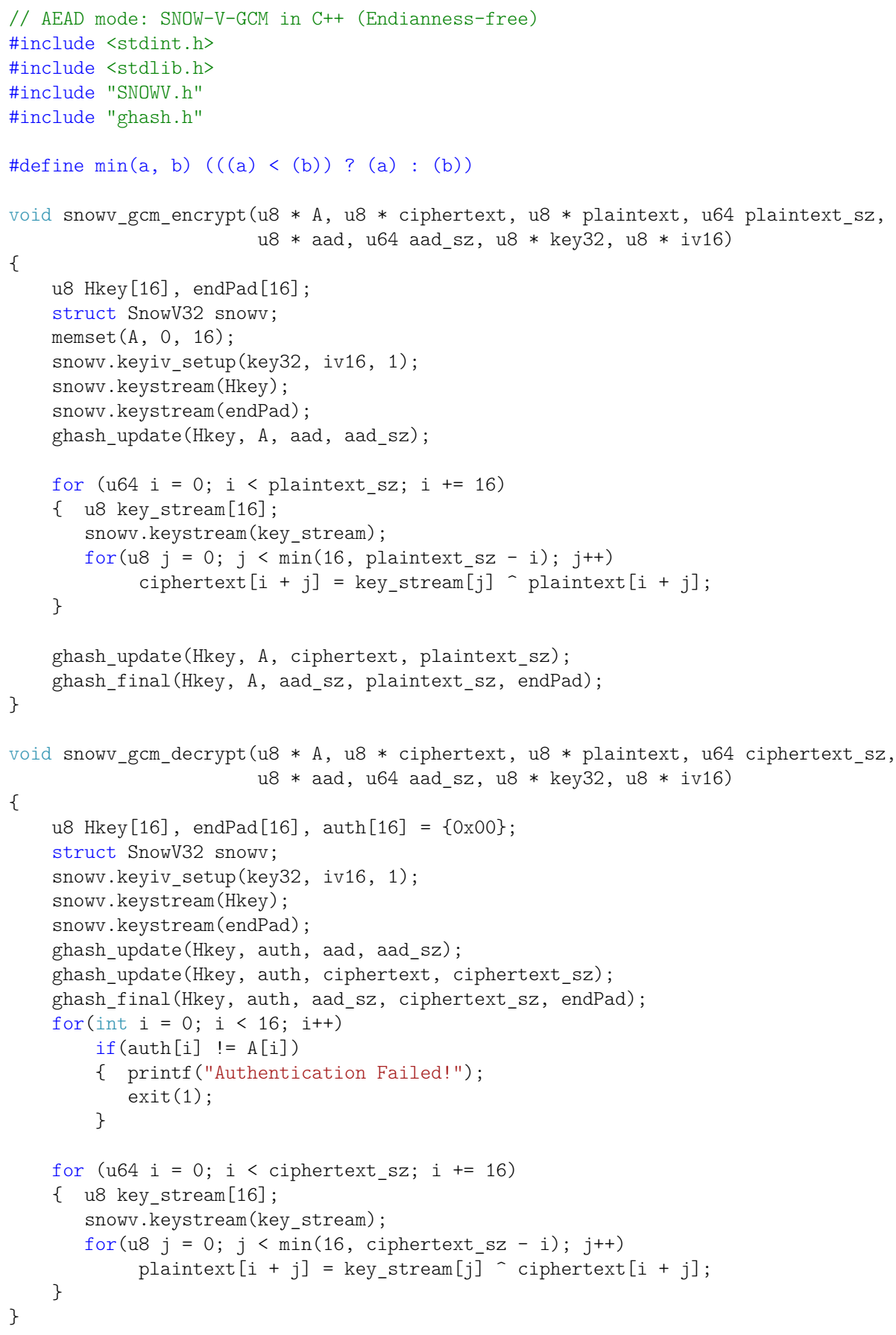




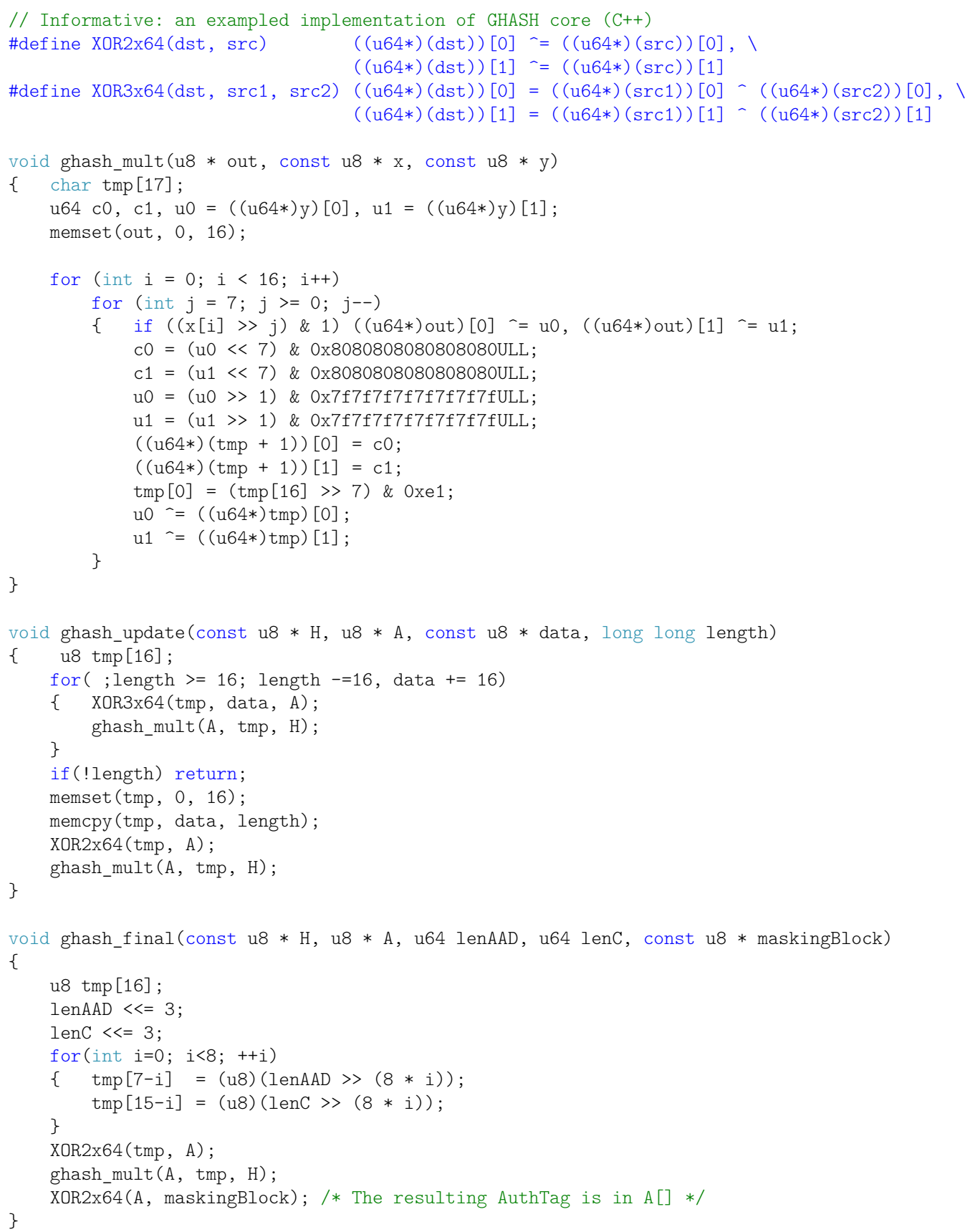




\section{E SNOW-V Reference Implementation with SIMD}

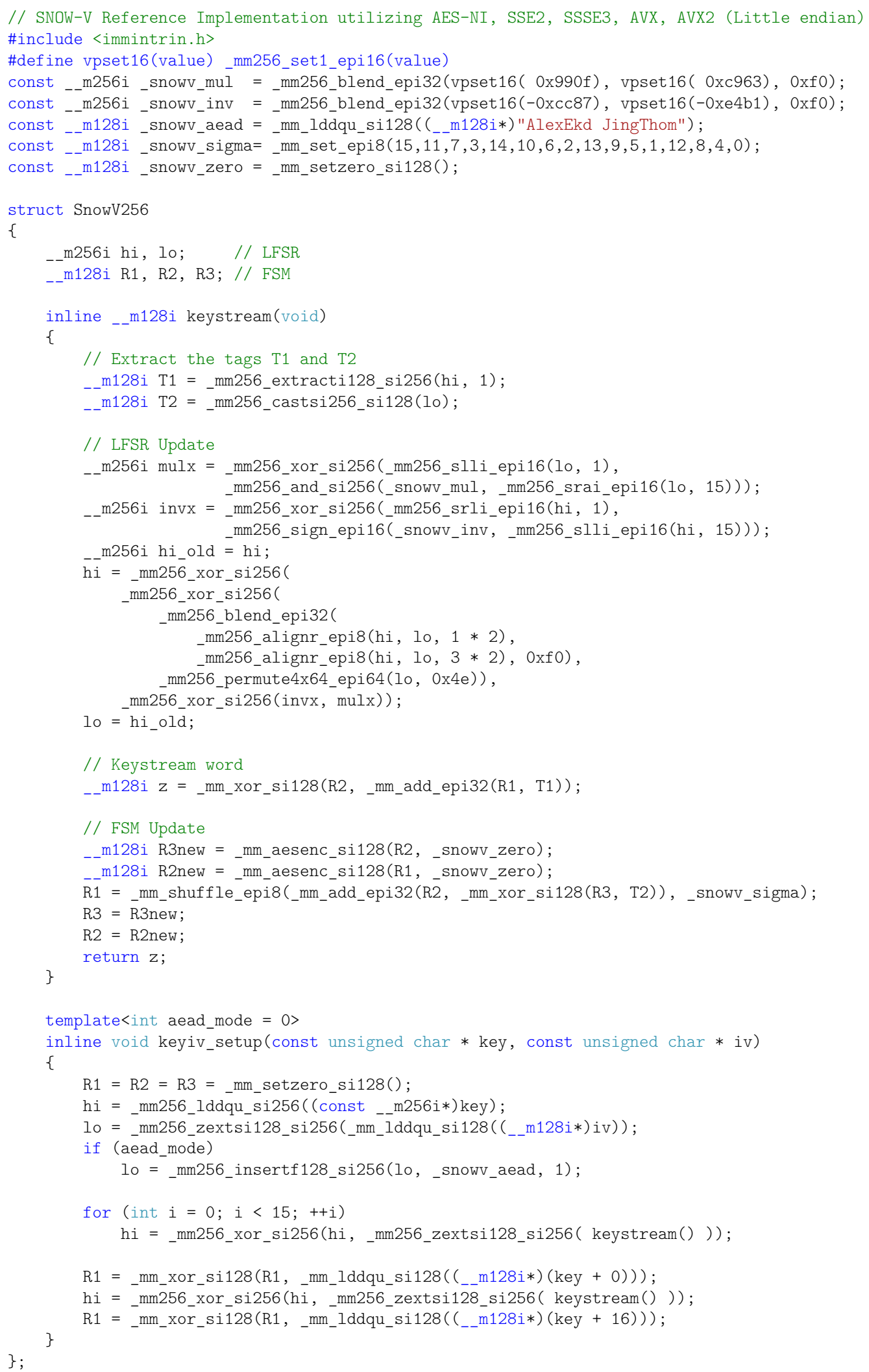




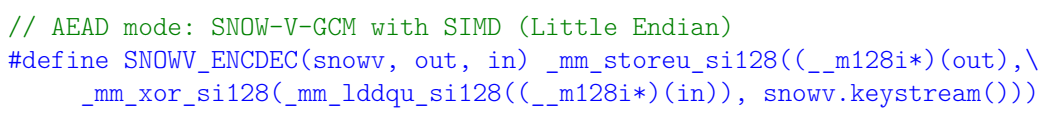




\section{F Hardware implementation aspects}

When designing new algorithms targeting existing systems, reusability of hardware components is important to reduce area and cost of the ASICs. Many systems dealing with network communication security implement some form of AES acceleration, either in a specialized ASIC or as specialized CPU instructions. SNOW-V leverages this co-existence by using two full AES encryption rounds as the main nonlinear element. A hardware implementation of SNOW-V can utilize either one or two external AES cores, if present, or implement its own AES encryption rounds in a stand-alone design for maximum speed. Although a 128-bit implementation is straight-forward from the algorithm description, it has some drawbacks when we only have one single external AES core available, as is the case in many constraint implementations. In this section we will consider how to implement SNOW-V using a single AES core with a 64-bit hardware architecture. We will refer to the 64-bit and 128-bit hardware implementations as the 64-SNOW-V and 128-SNOW-V respectively.

\section{F.1 SNOW-V 64-bit Hardware Architecture}

In this section we propose a 64-bit hardware architecture where SNOW-V requires a single AES encryption core (external or built-in), and each clocking of 64-SNOW-V produces 64 bits of the keystream.

Cons: additional two 64-bit delay registers $D 1$ and $D 2$ are needed; the logic needs additional 6 64-bit multiplexers; two clocks to produce 128 bits of keystream that actually halves the speed.

Pros: a single AES encryption core is needed; produces 64 bits of keystream at each clock; all basic operations in both FSM and LFSR, such as XOR and ADD, are now halved in size.

In order to utilize a single AES core the FSM update function should be split into two steps. The main critical path is the AES EncRound, which means that while splitting FSM into two stages we should avoid any extra logic on the input and output signals of the AES core. Thus, input to and output from the AES core must be registers.

Let us split all 128-bit registers and all 128-bit signals of the FSM block, say $X$, into two 64-bit halves as $X_{a}$ (low) and $X_{b}$ (high). We also assume that the tap values $T 1$ and $T 2$ from the LFSRs also arrive in 64 -bit chunks, such that every even clock FSM gets $T 1_{a}$ and $T 2_{a}$, and every odd clock $T 1_{b}$ and $T 2_{b}$.

In Figure 7 we propose a possible way to split the FSM such that it contains the two circuits for even and odd steps, 0 and 1 resp. (excluding the gates needed for initialization). One can notice that after these two steps the content of the registers $R 1, R 2, R 3$ become updated to new 128 -bit values ' $R 1,{ }^{\prime} R 2,{ }^{\prime} R 3$, and ready to process the next 128 bits of data with the same two steps. The above two circuits are then combined into a single circuit using multiplexers.

In Figure 8 the complete hardware architecture for 64 -bit SNOW-V is presented. There are 7 64-bit multiplexers in total, and we denote the control signal to them by $M_{1} . . M_{7}$, respectively. There are also 5 64-bit AND gates, the purpose of which is to either bypass the signal or block it. Those AND blocks are controlled by four signals $G_{A}, G_{Z}, G_{K}, G_{F}$, the latter controls 2x64 AND-blocks. The Control Unit in Figure 8 generates the control signals for the multiplexers and AND gates depending on the state of SNOW-V.

Critical path. Our primary assumption is that the AES encryption round would be the main critical path $(M C P)$. However, one can easily determine that the secondary critical path $(S C P)$ would be the sequence AND-MUX-XOR-ADD-XOR-MUX over 2x32-bit integers, denoted by red wires in Figure 8 . Thus, when selecting 32-bit adders one should make sure that they are fast enough so that the $M C P$ is sustained.

The algorithm has 3 stages: 


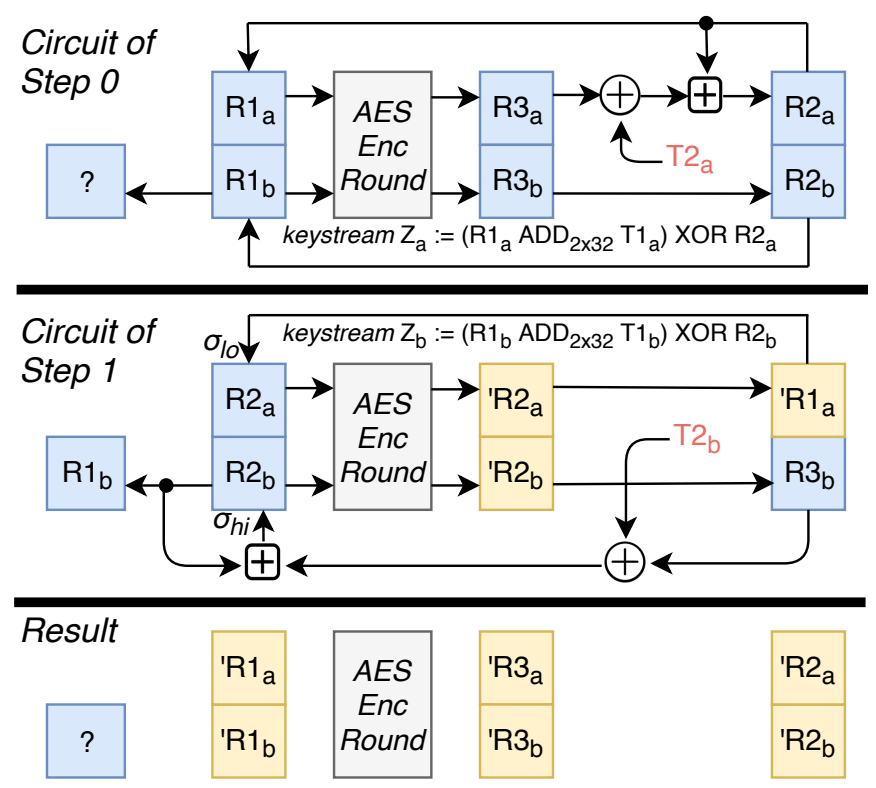

Figure 7: Splitting of FSM into two steps in order to utilize only one AES core (excluding initialization steps).

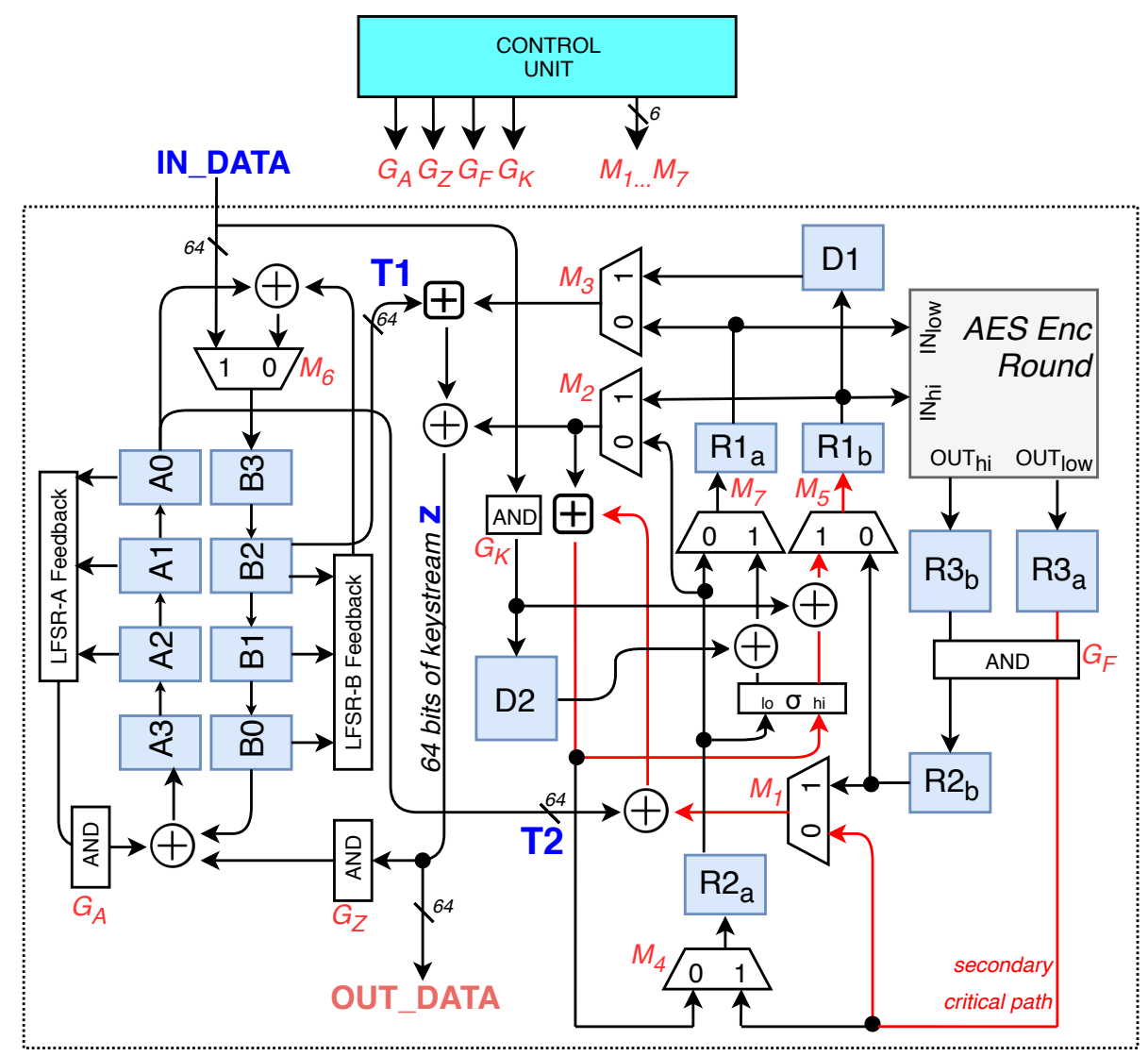

Figure 8: Hardware architecture of 64-bit SNOW-V with a single AES core. 
Stage 1 - Loading. The design is constructed in such a way that the registers do not need to have any RESET signal. Instead, all registers will be sequentially loaded with the key and IV, and the remaining registers will be zeroized, during this stage.

The stage begins with a strobe signal on LOAD, and the first 64-bit chunk of data is expected on the IN_DATA bus. In total, the stage expects to receive 864 -bit words each clock in the following order: $\left\{i v_{0}, i v_{1}, k_{0}, k_{1}, 0,0, k_{2}, k_{3}\right\}$.

In this stage, the control unit should block AND gates $G_{Z}=G_{A}=0$, and set $M_{6}=1$, in order to concatenate LFSRs A and B into a single large LFSR while shifting in the initialization data. In order to zeroize FSM registers, the control unit should block $G_{F}=0$ and also enforce the multiplexer inputs $M_{4}=1, M_{5}=M_{7}=0 . G_{K}$ is set to 0 .

After the 8 clocks where the key and IV are loaded, we proceed to stage 2 .

Stage 2 - Initialization. In this stage, the FSM works in the same way as when it produces keystream output symbols, i.e. the multiplexer control signals switches according to even/odd clock cycle as explained previously. The LFSRs are connected together by setting $G_{Z}=G_{A}=1$ and switching $M_{6}=0$ to disable any external input.

Note that we placed the AND gating after the registers $R 3_{a}, R 3_{b}$, so that we do not add extra depth to the critical path of AES core, hence these registers will not be zeroized. To overcome this problem the control unit generates $G_{F}=0$ in the first clock of this stage, and then sets $G_{F}=1$ until the end of stage 2 . We keep $G_{K}=0$ for the first 28 clocks. In the remaining 4 clocks we need to XOR the key $K$ to $R 1$ according to the initialization procedure. So we enable $G_{K}=1$ and expect to receive $\left\{k_{0}, k_{1}, k_{2}, k_{3}\right\}$ consecutively from the input bus IN_DATA. After this, the circuit is ready to produce keystream words.

Stage 3 - Keystream generation. Both LFSR and FSM operate normally. The control unit in this stage detaches the $Z$ signal from being feeded into LFSR-A by setting $G_{Z}=0$. The input bus is also detached by setting $G_{K}=M_{6}=0$.

\section{F.2 Theoretical Analysis of 64/128-bit SNOW-V in Hardware}

The area will be estimated in terms of gate equivalence $(\mathbf{G E})$, where $1 \mathrm{GE}=$ size of a NAND gate. The speed will be estimated in terms of Gigabits per second (Gbps), based on known speed results of AES circuits. We will use GE values given in [Sam00] for 1-speed technology elements.

For comparison with AES, we will use one of the more recent results from [UMHA16] where an area-speed optimized AES-128 (10 rounds) on NanGate 15nm technology runs with the speed 71.19 Gbps and has the area 17232 GE. This means that having the same design, AES-256 (14 rounds) would run with the speed of 50.85 Gbps.

Our basic assumption is that the AES core is the critical path of the SNOW-V circuit. Thus, if SNOW-V would utilize a single AES core as above, the speed of 64-SNOW-V could be as high as $\mathbf{3 5 6}$ Gbps. The speed of 128-SNOW-V with two AES cores is therefore as high as 712 Gbps. What remains is to calculate the hardware cost of SNOW-V, excluding the external AES core, but including the cost of integration into that external AES core. We will also exclude the control unit, as this can be implemented with a very few gates and latches and every implementation will have slightly different needs of control and ready signaling.

State Registers. For 64-SNOW-V, there are 512 registers for the LFSR and 6x64+2x64 registers for the FSM. Since our 64-bit implementation does not require complex latches (e.g., no RESET), we can use the simplest D-latch with Q-output only from [Sam00] [FD1Q]. The total cost is $1024 * 4.33=\mathbf{4 4 3 4} \mathbf{~ G E}$.

For 128-SNOW-V we also need 512 registers for the LFSRs without reset, and 3x128 registers with RESET [FD2Q], thus resulting in $512^{*} 4.33+\left(3^{*} 128\right)^{*} 5.67=4394 \mathbf{G E}$.

For arithmetical 32-bit adders we suggest to take, for example, a Han-Carlson 32-bit adder, as it has a low area overhead (15\%-25\% larger than Ripple-Carry adders) and a very small delay $O(\log (n))$ - which is important in order to keep the critical path upper 
bounded by the AES round function. We can estimate these components as 4x(30FADD3 +2 HADD 2$)+20 \%=4(30 * 6.33+2 * 3.67) * 1.20=\mathbf{9 4 7} \mathbf{~ G E}$ for $64-\mathrm{SNOW}-\mathrm{V}$ and $1894 \mathbf{~ G E}$ for $128-\mathrm{SNOW}-\mathrm{V}$.

The remaining part of the FSM update logic therefore contains $3 \times 64$ AND2 + $6 \mathrm{x} 64 \mathrm{MUX} 2+4 \mathrm{x} 64 \mathrm{XOR} 2=3 * 64 * 1.33+6 * 64 * 2.33+4 * 64 * 2.33=1747$ GE for $64-\mathrm{SNOW}-\mathrm{V}$ and $(128 \mathrm{AND} 2+3 \mathrm{x} 128 \mathrm{XOR} 2)=\mathbf{1 0 6 5} \mathbf{~ G E}$ for $128-\mathrm{SNOW}-\mathrm{V}$.

LFSR Update logic involves two circuits for the feedback functions. 16-bit field multiplications by $\alpha, \alpha^{-1}, \beta, \beta^{-1}$ can be done with 8 XORs in each case, since the Hamming weight of both $g^{A}(\alpha)$ and $g^{B}(\beta)$ is 8 .

However, let us have a closer look on how each bit of, e.g. $a_{16}$ is calculated. Each bit $a_{16}[i], 14 \geq i \geq 1$ is unconditionally depending on four bits, namely

$$
a_{16}[i]: a_{0}[i-1]+a_{1}[i]+a_{8}[i+1]+b_{0}[i]
$$

The end bits are easy to work out too. Some of the bits of $a_{16}$ are also depending on $a_{0}[15]$ and $a_{8}[0]$, due to the multiplication with $\alpha$ and $\alpha^{-1}$. Table 5 gives a full overview of the dependencies for both $a_{16}$ and $b_{16}$.

Table 5: Bit dependencies due to multiplications for $a_{16}$ and $b_{16}$.

\begin{tabular}{|l|c|c|c|c|c|c|c|c|c|c|c|c|c|c|c|c|c|}
\hline $\mathrm{i}$ & 15 & 14 & 13 & 12 & 11 & 10 & 9 & 8 & 7 & 6 & 5 & 4 & 3 & 2 & 1 & 0 & Depending on \\
\hline \multirow{2}{*}{$a_{16}[i]$} & $\checkmark$ & & & $\checkmark$ & $\checkmark$ & & & $\checkmark$ & & & & & $\checkmark$ & $\checkmark$ & $\checkmark$ & $\checkmark$ & $a_{0}[15]$ \\
& $\checkmark$ & $\checkmark$ & & & $\checkmark$ & $\checkmark$ & & & $\checkmark$ & & & & & $\checkmark$ & $\checkmark$ & $\checkmark$ & $a_{8}[0]$ \\
\hline \multirow{2}{*}{$b_{16}[i]$} & $\checkmark$ & $\checkmark$ & & & $\checkmark$ & & & $\checkmark$ & & $\checkmark$ & $\checkmark$ & & & & $\checkmark$ & $\checkmark$ & $b_{0}[15]$ \\
& $\checkmark$ & $\checkmark$ & $\checkmark$ & & & $\checkmark$ & & & $\checkmark$ & & $\checkmark$ & $\checkmark$ & & & & $\checkmark$ & $b_{8}[0]$ \\
\hline
\end{tabular}

This means that in order to compute $a_{16}[i]$, we have to XOR 4,5 , or 6 different input bits. For example, in the table above we see that the $a_{16}[13]$ is only dependent on the basic input bits in Equation 13, and the XOR gate needs 4 inputs:

$$
a_{16}[13]=a_{0}[12]+a_{1}[13]+a_{8}[14]+b_{0}[13] .
$$

On the other hand, $a_{16}[11]$ needs to XOR 6 inputs:

$$
a_{16}[11]=a_{0}[10]+a_{1}[11]+a_{8}[12]+b_{0}[11]+a_{0}[15]+a_{8}[0] .
$$

since the multiplication with $\alpha$ and $\alpha^{-1}$ will both influence that bit.

Following the hardware architecture of 64-SNOW-V given in Figure 8 we have to split the calculation of the feedback function LFSR-A due to the control AND-gateway. Also, the circuit should compute 4 16-bit updates in parallel. Summarizing, we get (a) LFSR-A feedback function, excluding input from $b_{0}: 4 \mathrm{x}(5 \mathrm{XOR} 3+6 \mathrm{XOR} 4+5 \mathrm{XOR} 5)$ $\approx 4 *(5 * 4.00+6 * 6.00+5 * 8.00)=\mathbf{3 8 4} \mathbf{G E} ;$ (b) LFSR-B feedback function, including input from $a_{0}: 4 \mathrm{x}(4 \mathrm{XOR} 4+8 \mathrm{XOR} 5+4 \mathrm{XOR} 6) \approx 4 *(4 * 6.00+8 * 8.00+4 * 10.00)$ $=\mathbf{5 1 2} \mathbf{G E}$; (c) the remaining part of LFSR block: 2x64AND2 + 64XOR3 + 64MUX2 $=64 *(2 * 1.33+4.00+2.33)=\mathbf{5 7 5}$ GE. For 128 -SNOW-V we simply double the above numbers.

Integration into an external AES Engine requires input multiplexers for 128 bits of the plaintext and 128 bits for the round key. However, the AES round keys $C 1$ and $C 2$ are zeroes so that we can use $128 \mathrm{AND}$ gates, instead. In total we get $128 \mathrm{MUX} 2+$ $128 \mathrm{AND} 2=128 *(2.33+1.33)=\mathbf{4 6 8} \mathbf{~ G E}$ for $64-\mathrm{SNOW}-\mathrm{V} .128-\mathrm{SNOW}-\mathrm{V}$ requires two such integration circuits.

In case we decide to implement SNOW-V with its own internal AES EncRound, the hardware cost could be as small as 16 AES SBoxes, plus some logic for MixColumn. Also note that in this case the critical path decreases since we only need the forward SBox 
and thus any outer multiplexing logic for a combined forward and inverse SBox can be removed. This could lead to a potential speed up for 128-SNOW-V.

The part MixColumn of AES encryption round, applied to the AES state $\left\{r_{i, j}\right\}$ for $0 \leq i, j \leq 3$, is the following matrix multiplication.

$$
\left[\begin{array}{l}
r_{0, j}^{\prime} \\
r_{1, j}^{\prime} \\
r_{2, j}^{\prime} \\
r_{3, j}^{\prime}
\end{array}\right]=\left[\begin{array}{llll}
2 & 3 & 1 & 1 \\
1 & 2 & 3 & 1 \\
1 & 1 & 2 & 3 \\
3 & 1 & 1 & 2
\end{array}\right] \cdot\left[\begin{array}{l}
r_{0, j} \\
r_{1, j} \\
r_{2, j} \\
r_{3, j}
\end{array}\right], 0 \leq j \leq 3
$$

That can be computed in a depth 2 circuit, for each $0 \leq j \leq 3$, as $t_{0}=r_{0}+r_{1}$, $t_{1}=r_{1}+r_{2}, t_{2}=r_{2}+r_{3}, t_{3}=r_{3}+r_{0}$, and then $r_{0}^{\prime}=2 t_{0}+t_{2}+r_{1}, r_{1}^{\prime}=2 t_{1}+t_{3}+r_{2}$, $r_{2}^{\prime}=2 t_{2}+t_{0}+r_{3}, r_{3}^{\prime}=2 t_{3}+t_{1}+r_{0}$, where multiplication $2 t_{i}$ is the multiplication by $x$ in the Rijndael field and can be implemented with 3XOR2. The cost of MixColumn is therefore $4 \mathrm{x} 4 \mathrm{x}(8 \mathrm{XOR} 2+8 \mathrm{XOR} 3+3 \mathrm{XOR} 2)=\mathbf{9 2 2} \mathbf{G E}^{3}$.

The cost of a single forward SBox is around $220 \mathrm{GE}$ (see, e.g., [RMTA18]). Thus, for a single internal AES EncRound the total cost is $16 * 220+922=\mathbf{4 4 4 2}$ GE. Summarizing the above we can derive the comparison given in Table 6 .

Table 6: Theoretical comparison of four SNOW-V versions vs AES-256 in hardware.

\begin{tabular}{|c|c|c|c|c|c|}
\hline $\begin{array}{c}\text { Hardware } \\
\text { design }\end{array}$ & $\begin{array}{c}\text { AES-256 } \\
\text { from [UMHA16] }\end{array}$ & $\begin{array}{c}\text { 64-SNOW-V } \\
\text { 1xAES } \\
\text { ext. core }\end{array}$ & $\begin{array}{c}\text { 64-SNOW-V } \\
\text { 1xAES } \\
\text { int. round }\end{array}$ & $\begin{array}{c}\text { 128-SNOW-V } \\
\text { 2xAES } \\
\text { ext. cores }\end{array}$ & $\begin{array}{c}\text { 128-SNOW-V } \\
\text { 2xAES } \\
\text { int. rounds }\end{array}$ \\
\hline \hline Area & $17232 \mathrm{GE}$ & $9067 \mathrm{GE}$ & $13041 \mathrm{GE}$ & $11231 \mathrm{GE}$ & $19179 \mathrm{GE}$ \\
Speed & $50.85 \mathrm{Gbps}$ & $358 \mathrm{Gbps}$ & $358+$ Gbps & $712 \mathrm{Gbps}$ & $712+\mathrm{Gbps}$ \\
\hline
\end{tabular}

\footnotetext{
${ }^{3}$ Recent results in [Max19] suggest that MixColumn can be implemented with 4x92 gates. However, we believe that the proposed classical solution with $4 \times 108$ gates is a better choice since it has a lower depth of the critical path, thus allowing SNOW-V to perform faster.
} 